\title{
Cavitation dans les pompes
}

\section{Cavitation in pumps}

\author{
A report of Cavitation Working Group No. 2 of Société Hydrotechnique de France
}

PAR P. GUITON,

INGÉNIEUR A LA MAISON L. BERGEHON

\begin{abstract}
A la demande de la Commission de la Cavitation, le rapport fait le point actuel des connaissances que l'on peut considérer comme acquises concernant la cavitation dans les pompes et remplit les buts suivants:

$1^{\circ}$ Il rassemble, d̀ l'usage des constructeurs et des utilisateurs de turbines, l'essentiel des renseignements sur cette question afin de leur permettre d'en tirer des remarques utiles pour leurs propres problèmes de cavitation. $2^{\circ}$ Il constitue l'ontil nécessaire anx travaux ultérieurs du Groupe sur la cavitation dans les pompes et pour cela

a) Crée un cadre de chapitres et de paragraphes où tonte question ultérieure et tout renseignement viennent trouver leur place;

b) Précise le langage commun de façon que les définitions et les notations adoptées évitent les confusions ultérieures, notamment en ce qui concerne les différents stades de cavitation;

c) Etablit un diagrammie général de représentation permettant la confrontation des résultats. $3^{\circ}$ Il présente à l'aide de ce diagramme quelques résultals tỵpes déduits d'une première statistique et les compare avec les formules et abaques classiques.
\end{abstract}

At the request of the Cavitation Commission this report surveys the present state of es tablished knowledge of cavitation in pumps and fulfils the following aims:

1. It provides a summary of essential information on this question for turbine constructors and asers so that they maly draw on this source for the solation of their own cavitation problems.

2. It forms an essential tool for the further work of the Group on cavitation and in this respect:

a) it lays down a framework of chapters and paragraphs thus providing a place for all further questions and information;

b) it defines a common language of definitions and notations to avoid later confusion, especially with respect to the different stages of cavitation;

c) it establishes a general representative graph to allow the results to be compared.

3. By means of this graph it presents some typical results deduced from the first statistical study and compares them wilh conventional formulas and graphs.

Ce texte a été élaboré dans le cadre de la Commission de la Cavitation de la Société Hydrotechnique de France par le Groupe de travail $\mathrm{n}^{\circ} 2$, dont la composition était la suivante : MM. Guiton, rapporteur du Groupe, Maison L. Bergeron; Bovchon, Société Rateau; Huguenin, Laboratoire d'Hydraulique et de Cavitation de l'Ecole Nationale d’Ingénieurs Arts et Métiers de Paris; Manon, Société Le Matériel Electrique SW; de Saint-Vaulry, Compagnie de Construction Mécanique, procédés Sulzer; Sasia, Société Worthington; et le professeur Tenot.

\section{CHAPITRE 1}

\section{GENÉRALITÉS ET NOTATIONS}

\section{i.1. Généralités.}

\subsubsection{Comparaison ayec les turbines.}

Par définition, il existe un phénomène de cavitation dans une pompe, comme dans toute machine ou appareil hydraulique, quand il $\mathrm{y} a$, dans le liquide intérieur, des cavités de vapeur, si petites soient-elles.
Mais les pompes et les turbines présentent, à ce sujet, bien des différences. Il a paru intéressant de le rappeler, tout d'abord, pour ceux des membres de la S.H.F. qui sont plus familiarisés avec les turbines qu'avec les pompes. Parmi ces différences, les principales sont les suivantes:

1.1.1.1. Pression de vapeur. - Comme on le sait pour que des cavités se forment, il faut que la pression absolue la plus faible à l'intérieur de la machine, $p$ minimale, s'abaisse jusqu'à attein- 
dre la pression de vapeur $p_{v}$ du liquide dans la pompe. Contrairement à ce qui a lieu généralement pour les turbines, $p_{v}$ est souvent loin d'être négligeable (pompage d'eau chaude, hydrocarbures etc.). Nous reviendrons plus loin, au chapitre 8 , sur les conséquences qui en résultent.

1.1.1.2. Cavitation à l'entrée. - La traversée de la roue de la pompe (ou de la première roue s'il s'agit de pompes multicellulaires) mettant rapidement le liquide en pression, la zone de pression minimale de la pompe se situe normalement dans cette roue, très près en aval de l'entrée dans celle-ci. (Nous mettrons de côté pour l'instant les cavitations se produisant ailleurs que dans l'œillard de la première roue, qui feront l'objet du chapitre 6.)

La cavitation de la pompe, pour un débit et une vitesse de rotation donnés, dépend donc avant tout des conditions d'aspiration et de charge existant à l'entrée de la première roue. Elle est, par contre, pratiquement indépendante des conditions existant à la sortie de la roue.

C'est donc l'inverse de ce qui a lieu pour les turbines.

1.1.1.3. Cavitation accentuée, en général inacceptable. - En se développant à l'entrée de la roue, la cavitation perturbe très rapidement tout l'écoulement dans celle-ci, altérant très vite de façon importante les caractéristiques de fonctionnement. Contrairement aux turbines, il n'est donc guère possible, en général, d'admettre le fonctionnement en cavitation d'une pompe.

1.1.1.4. Etude systématique des érosions difficiles. - Les érosions par cavitation sont par suite presque toujours plus lentes que pour les turbines, et le seuil de cavitation industrielle (voir 1.1.2.) correspondant à la limite d'emploi, est en général hxé par une chute de hauteur, de débit ou de rendement et non par une rapidité d'érosion comme cela a souvent lieu pour les turbines.

A cause de cela, et sauf quelques cas particuliers, les observations systématiques des zones d'érosion sont beaucoup plus rares que pour les turbines et le groupe de travail n'en a guère à présenter actuellement.

Cette lacune tient aussi du fait que les pompes, combien vitales pourtant, ne sont le plus souvent que des auxiliaires et ćchappent rapidement aux investigations de l'exploitant qui, en sénéral, ne demande qu'à les oublier : autre différence encore entre les turbines et les pompes.

1.1.1.5. Bruits parfois inéluctables. - Les bruits de cavilation, qui sont la hantise des constructeurs de pompes, apparaissent souvent avant le seuil de cavitation industrielle (voir 1.1.6) qui limite généralement l'emploi de la machine.

Force est, le plus souvent, de les accepter, car, comme nous allons le voir, il n'y a pas ici, comme pour les turbines, la possibilité de faire entrer systématiquement de l'air dans la machine.

1.1.1.6. Remède de l'entrée d'air difficilement utilisable. - On sait qu'il est classique, pour les turbines, d'atténuer le bruit de cavitation en laissant rentrer systématiquement de l'air dans l'œillard de la roue.

Or, dans une pompe, une petite rentrée d'air étouffe bien le bruit. Mais elle affecte rapidement les caractéristiques de fonctionnement de la machine: l'air étant entraîné au travers de la roue, le fluide traversant celle-ci devient compressible, ce qui modifie sa mise en pression.

1.1.1.7. Dépression du liquide souvent en amont de la machine. - Alors que, pour les turbines, la zone de dépression ne remonte pas en amont de la roue, pour les pompes, elle peut s'étendre très loin en amont de celle-ci, dans beaucoup d'installations.

Il peut y avoir alors bien avant la pompe un début de dégazage qui tend à favoriser l'apparition de la cavitation.

1.1.1.8. Parties de la machine pouvant être touchées par la cavitation en aval de la $1^{\text {re }}$ roue. - Alors que, pour les turbines, il n'y a guère de cavitation en dehors de la roue et de l'aspirateur, pour les pompes, loin de se limiter à la $1^{\text {re }}$ roue, les parties de la machine touchées par la cavitation peuvent comprendre les diffuseurs, les redresseurs, etc., et enfin des roues autres que la $1^{\text {re }}$ roue, dans le cas de pompes multicellulaires.

\subsubsection{Cavitation PHYSique e't Cavitation indus- TRIELLE.}

1.1.2.1. Cavitation physique. - Nous dirons qu'il y a cavitation physique dans la machine dès qu'il y apparaît les moindres cavités ou bulles de cavitation. On ne dit pas pour autant, industriellement, que la pompe cavite.

1.1.2.2. Cavitation industrielle. - Industriellement, on ne parle de cavitation que lorsque la cavitation physique est développée au point d'entrainer des perturbations nuisibles du fonctionnement de la machine (voir 2.2.2)

\subsection{Notations.}

\subsubsection{Catégories de paramètres : Paramìtres EXTERNES ET PARAMĖTRES INTERNES.}

Les paramètres intervenant dans l'étude de la cavitation peuvent être répartis en deux catégories : 
Première catégorie - Paramètres externes: ceux qui ont trait au comportement extérieur de la machine et auxquels on est limité le plus souvent en l'absence de renseignements sur son dimensionnement interne.

Deuxième catégorie - Paramètres internes: ceux faisant intervenir le dimensionnement interne et en particulier le diamètre $D_{a}$ de l'œillard de la première roue.

Les premiers intéressent surtout le projeteur et l'utilisateur et les seconds le constructeur.

\subsubsection{Principalx paramétres externes.}

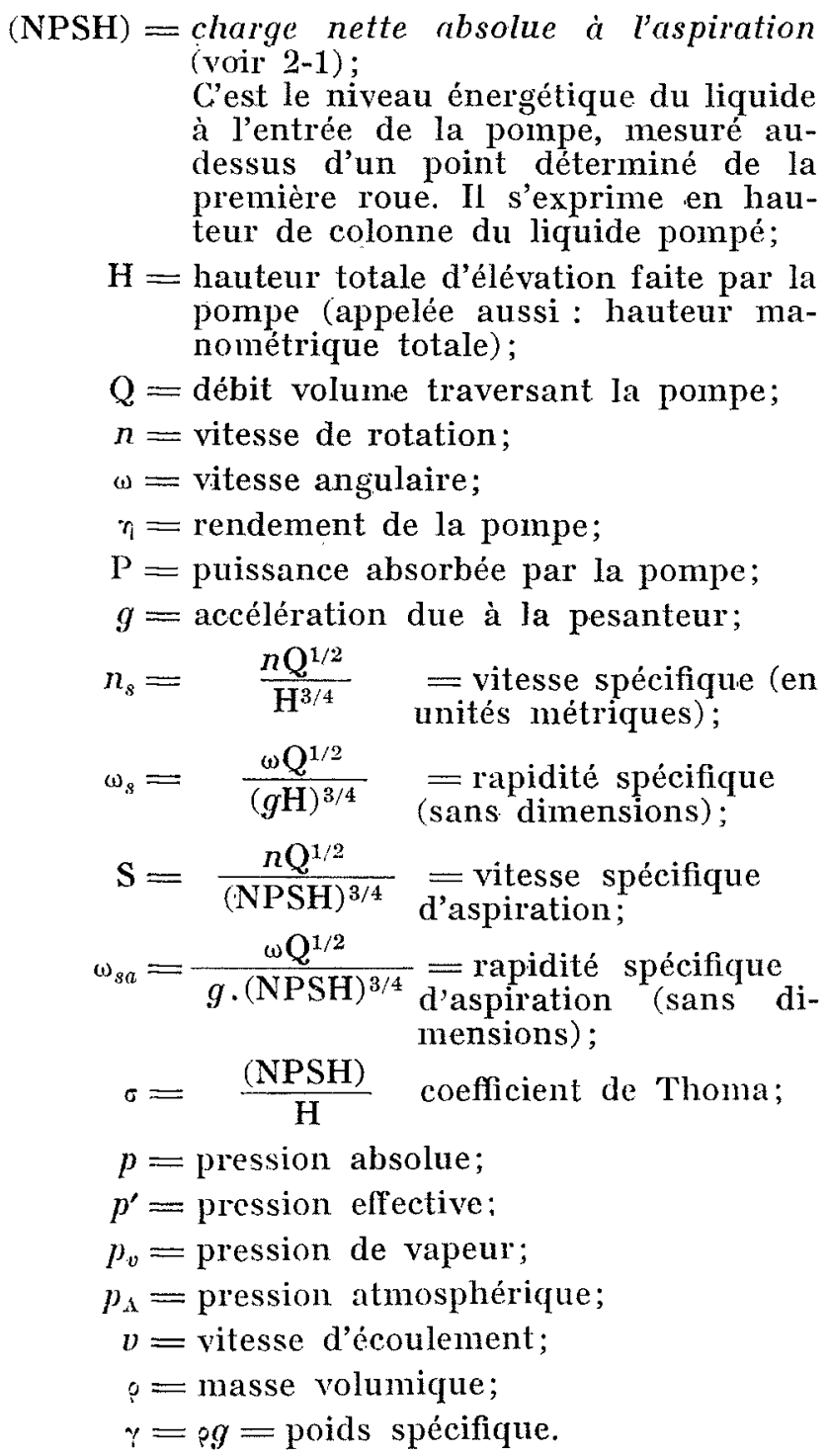

\subsubsection{PRINCIPAUX PARAMÈTRES INTERNES.}

$\mathrm{D}_{a}=$ diamètre d'entrée de la roue;

$D_{r}=$ diamètre de sortie de la roue;

$\mathrm{S}_{a}=$ aire de la section utile d'entrée de la roue; $m=$ coefficient d'obstruction de l'œillard;

$u_{a}=$ vitesse d'entrainement au diamètre $\mathrm{D}_{a}$;

$v_{a}=$ vitesse axiale moyenne dans la section $\mathrm{S}_{a}$;

$\psi_{a}=\frac{(\mathrm{NPSH})}{u_{u}{ }^{2} / 2 g} \quad \begin{gathered}=\text { coefficient de charge } \\ \text { nette absolue à l'aspira- }\end{gathered}$ tion;

$\varphi_{a}=v_{a} / u_{a} \quad \begin{gathered}=\text { coefficient de débit } \\ \text { d'aspiration; }\end{gathered}$

$k_{a}=\mathrm{NPSH} /\left(v_{a}^{2} / 2 g\right)$;

$\Omega_{a}=$ coefficient de rapidité spécifique $\dot{a}$ l'aspiration;

$\mathrm{S}^{\prime}=\frac{n(\mathrm{Q} / m)^{1 / 2}}{(\mathrm{NPSH})^{3 / 4}}=$ vitesse spécifique d'aspiration corrigée (en unités métriques);

$\omega_{a}^{\prime}=\frac{\omega(\mathrm{Q} / m)^{1 / 2}}{g^{3 / 4}(\mathrm{NPSH})^{3 / 4}}=$ rapidité spécifique

\subsubsection{LETTRES EN INDICE}

$a=$ caractérise la section d'entrée de la première roue de la pompe;

$o=$ caractérise la section de mesure à l'entrée de la machine;

$b=$ caractérise le stade de cavitation physique correspondant à la formation des premières petites cavités ou bulles:

$d=$ caractérise le stade de cavitation physique correspondant au point où la courbe $\mathrm{H}-\mathrm{Q} \dot{\mathrm{a}}$ vitesse constante commence à décoller de la courbe $\mathrm{H}-\mathrm{Q}$ en charge.

$c=$ caractérise le stade de cavitation physique correspondant au début de cavitation industrielle défini par une perte de $3 \%$ sur $\mathrm{H}$ pour un débit et une vitesse de rotation donnés, par rapport à sa valeur en charge;

$g=$ caractérise la cavitation globale correspondant à la chute à pic de la courbe caractéristique $\mathrm{H}-\mathrm{Q}$ à vitesse de rotation constante;

disp $=$ disponible $=$ caractérise le $(\mathrm{NPSH})$ existant à l'entrée d'une pompe pour le différencier de celui ci-après;

requis $=$ celui qui est nécessaire pour éviter la cavitation (au stade $b, d, c$, ou $g$ ).

\section{1:3. Formulaire (voir aussi 1.2 .2 et 1.2.3).}

$$
\begin{aligned}
& (\mathrm{NPSH})=\frac{p_{0}}{\gamma_{0}}+\frac{v_{0}^{2}}{2 g}-\frac{p_{v}}{\gamma_{0}} ; \\
& \omega_{s}=0,0190 n_{s} \quad \omega_{s a}^{\prime}=0,0190 \mathrm{~S}^{\prime} ; \quad \omega_{s a}=0,0190 \mathrm{~S}
\end{aligned}
$$




$$
\begin{aligned}
\omega_{s a} & =\frac{\omega_{\mathrm{s}}}{\sigma^{3 / 4}} \quad \mathrm{~S}=\frac{n_{s}}{\sigma^{3 / 4}} \\
\omega_{s a}^{\prime} & =\frac{\omega_{s i}}{m^{1 / 2}} \quad \mathrm{~S}^{\prime}=\frac{\mathrm{S}}{m^{1 / 2}} ; \\
\psi_{a} & =k_{a} \varphi_{a}^{2} ;
\end{aligned}
$$$$
\Omega_{a}=\frac{\omega_{s a}}{m^{1 / 2}\left(8 \pi^{2}\right)^{1 / 4}} \quad \Omega_{a}=\frac{1}{\varphi_{a} k_{a}^{3 / 4}} ;
$$

$$
\varphi_{a}=\frac{\mathrm{Q}}{(\pi / 8) m \omega \mathrm{D}_{a}^{3}} \quad \frac{\mathrm{D}_{a}}{2}=\sqrt[3]{\frac{\mathrm{Q}}{m \omega \varphi_{a}}} .
$$

\section{CHAPITRE 2}

\section{INFLUENCE DE LA CHARGE NETTE ABSOLUE A L'ASPIRATION (NPSH) SUR LES CARACTERISTIQUES DE FONCTIONNEMENT DES POMPES}

\subsection{Charge nette absolue à l'aspiration (NPSH).}

\subsubsection{DÉFINITION}

Les conditions d'aspiration ou de charge à l'entrée de la première roue sont déterminantes. Comme elles sont liées à celles existant à l'entrée de la pompe, il est nécessaire de définir celles-ci sans ambiguïté, quitte à rappeler ce qui est connu de tous.

Ce qui importe, c'est l'écart existant entre la pression absolue totale (statique et dynamique) poussant le liquide à l'entrée de la pompe, soit $p_{0}+p_{0}\left(v_{0}^{2} / 2\right)$, et la pression de vapeur $p_{v}$ dans la pompe.

$p_{0}$, $\rho_{0}$ et $v_{0}$ sont respectivement la pression absolue ramenée à l'altitude de la zone de cavitation, la masse volumique et la vitesse moyenne du liquide à l'entrée de la machine.

Pour éviter la cavitation, il suffit que cet écart soit supérieur à la dépression maximale due à l'écoulement dans la pompe.

Exprimée en hauteur de colonne de liquide pompé, de poids spécifique $\gamma_{0}=\rho_{0} g$, à la température $t_{0}$ existant dans la pompe, cette dépression $\Delta h$ ne dépend, pour une roue donnée, que du débit et de la vitesse de rotation.

Ceci amène à exprimer de la même façon l'écart en question, qui devient :

$$
\frac{p_{0}}{\gamma_{0}}+\frac{v_{0}^{2}}{2 g}-\frac{p_{\eta}}{\gamma_{0}}
$$

Cette expression s'appelle la charge nette absolue à l'aspiration (en anglais «net positive suction head», en abrégé NPSH).

\subsubsection{Symboles.}

Pour cette expression, les symboles $H_{n \pi}$ et $H_{c a}$ ont èté proposés en France il y a quelques années. Dans la littérature mondiale on utilise, entre autres, les symboles : $\mathrm{H}_{s v}, \mathrm{H}_{n s}$ et (NPSH).
Comme ce dernier symbole est le plus répandu, notamment parmi les usagers des pompes, il a été retenu ici, de préférence aux autres, en attendant une normalisation internationale.

On écrira : (NPSH), la parenthèse permettant de faire suivre le symbole d'indices ou d'exposants, et de le faire entrer dans une expression algébrique.

$$
\text { Done : }(\mathrm{NPSH})=\frac{p_{0}}{\gamma_{0}}+\frac{v_{0}{ }^{2}}{2 g}=\frac{p_{v}}{\gamma_{0}}
$$

Comme l'altitude de la zone de cavitation est souvent mal définie, par convention, le (NPSH) est déterminé, sauf indications contraires, en ramenant la charge absolue piézométrique $p_{0} / \psi_{0}$ à l'altitude du centre $\mathrm{C}_{a}$ de l'entrée de la roue. (Intersection de l'axe de rotation de la machine avec le plan passant par les extrémités amont des aubes) (voir fig. 1.)

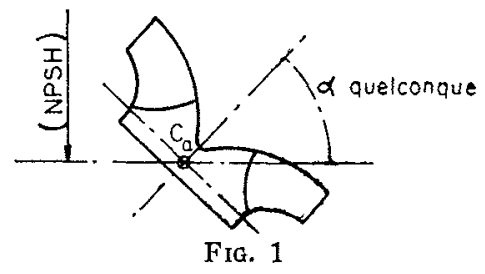

\subsection{3. $(\mathrm{NPSH})_{\text {disp. }}$ ET (NPSH) $)_{\text {requis. }}$}

On note $(\mathrm{NPSH})_{\text {disp. }}$ quand on veut indiquer qu'il s'agit du (NPSH) disponible, c'est-à-dire de celui existant effectivement à l'entrée de la pompe lors du fonctionnement considéré et non pas de celui nécessaire pour ne pas caviter, que l'on note (NPSH) requis.

Les (NPSH) correspondant aux divers stades de cavitation sont notés par les indices $b, d, c, g$ correspondant à ces stades, comme indiqué au paragraphe 2.5 ci-après (voir aussi 1.2.4).

\subsubsection{Détermination DE (NPSH) disp.}

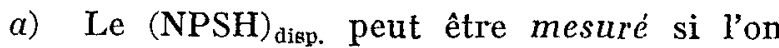
trouve une section de mesure convenable, en amont de l'entrée de la pompe; elle devra être, 


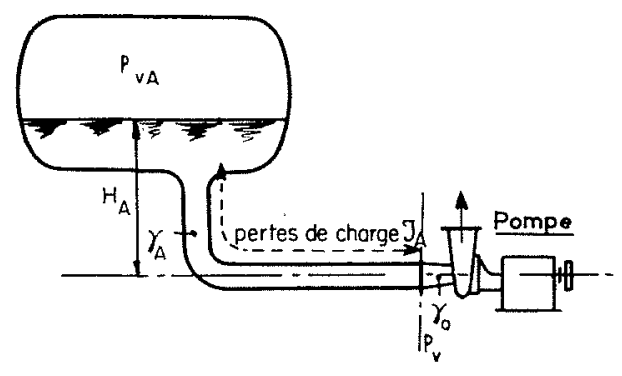

FIG. 2

notamment, exempte de prérotation. On devra en outre tenir compte de la perte de charge supposée entre cette section de mesure et la section d'entrée.

Dans ce cas, on mesure la pression effective $p^{\prime}{ }_{0}$ par rapport à l'atmosphère.

En prenant $p_{0}^{\prime}$ positif s'il s'agit d'une pression, l'entrée de la pompe étant en charge et en prenant $p_{0}^{\prime}$ négatif s'il s'agit d'un vide, on a alors, $p_{\mathrm{A}}$ étant la pression atmosphérique :

$$
\frac{p_{0}}{\gamma_{0}}=\frac{p_{A}+p_{0}^{\prime}}{\gamma_{0}}
$$

b) Le (NPSH) $)_{\text {disp. peut être aussi calculé à }}$ partir des données de l'installation, en particulier si le liquide pompé vient d'un réservoir ou d'un bassin comportant une surface libre du liquide (fig. 2 et 3 ).

En se référant aux figures, on a alors :

$$
(\mathrm{NPSH})_{\text {aisp. }}=\frac{p_{v A}+\gamma_{A}\left(\mathrm{H}_{\mathrm{A}}-\mathcal{Y}_{\mathrm{A}}\right)-p_{v}}{\gamma_{0}}
$$

avec les nolations suivantes:

$p_{v \mathrm{~A}}=$ pression totale (gaz + vapeur) régnant au-dessus de la surface libre;

$\gamma_{A}=$ poids volumique moyen du liquide dans la partie dénivelée de la tuyauterie;

$\mathrm{H}_{\mathrm{A}}=$ différence d'altitude entre la surface libre et le point $\mathrm{C}_{a}$ de la pompe, $\mathrm{H}_{\mathrm{A}}$ étant positif si l'entrée de la pompe est audessus de la surface libre, négatif si elle est en dessus;

$\mathcal{T}_{\mathrm{A}}=$ perte de charge entre le réservoir et la pompe évaluée en hauteur de colonne de liquide traversant la tuyauterie.

Par définition des pertes de charge, il $y$ a identité entre la valeur de (NPSH) disp. ainsi définie par (3) et celle définie précédemment par (1).

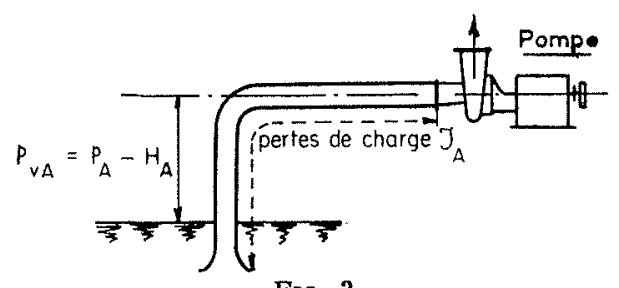

Fig. 3
En général, $\gamma_{A}=\gamma_{0}$, mais pour les pompes à eau chaude, par exemple, il peut se faire que les écarts de températures, entre la tuyauterie dénivelée et la pompe soient tels qu'ils ne permettent plus de confondre $\gamma_{A}$ et $\gamma_{0}$.

En ce qui concerne $p_{v A}$,

- s'il s'agit d'un réservoir en communication avec l'atmosphère extérieure ou d'un bassin à l'air libre, on a $p_{v \mathrm{~A}}=p_{\mathrm{A}}, p_{\mathrm{A}}$ étant la pression atmosphérique;

-.. s'il s'agit d'une enceinte fermée où règne uniquement la pression de vapeur du liquide pompé et si l'écart de température entre le liquide dans l'enceinte et celui dans la pompe est faible, on a $p_{v_{\mathbf{A}}}=p_{v}$.

C'est le cas, par exemple des pompes d'extraction de condenseur des pompes alimentaires et de certaines pompes à hydrocarbures.

\subsection{Influence d'une baisse de (NPSH) disp sur les caractéristiques de fonctionnement d'une pompe.}

\subsubsection{Courbes : $\mathrm{H}=f \quad\left[(\mathrm{NPSH})_{\text {disp. }}\right]$ $\mathrm{P}=f\left[(\mathrm{NPSH})_{\mathrm{disp} .}\right]$ FT $\eta=f\left[(\mathrm{NPSH})_{\mathrm{disp} .}\right]$ A $n$ ET $Q$ constants.}

Les figures $4 a, 4 b, 4 c$ et $4 d$ donnent schématiquement l'allure de la variation de hauteur $H$, de puissance absorbée $P$ et le rendement $\eta$, quand pour une pompe donnée, à une vitesse de rotation donnée, on fait baisser le (NPSH) disp. tout en maintenant le débit constant.

Ces figures sont tracées avec $(\mathrm{NPSH})_{\mathrm{disp}}$. en abscisse et $\mathrm{H}, \mathrm{P}$ et $\eta$ en ordonnées.

La figure $4 a$ a trait à une pompe centrifuge de rapidité š̉pécifique (*) relativement faible, soit

( () Conformément au vou de la Commission de la Cavitation, le Groupe de travail $n^{\circ} 2$ a adopté, pour caractériser la « vitesse spécifique» de la machine, le nombre sans dimension $: \frac{\omega Q^{1 / 2}}{\left(g \mathrm{H}^{3 / 1}\right.}$ avec $\omega=$ vitesse angulaire, $\mathrm{Q}=$ débit, $g$ =accélération due à la pesanteur, $\mathrm{H}=$ hauteur totale d'élévation, au lien des grandeurs habituellement utilisées pour les pompes :

$$
n_{s}=\frac{n \mathrm{Q}^{1 / 2}}{\mathrm{H}^{3 / 4}} \text { et } n_{s_{1}}=3,65 \frac{n \mathrm{Q}^{1 / 2}}{\mathrm{H}^{3 / 4}}=\frac{n(1000 \mathrm{QH} / 75)^{1 / 2}}{\mathrm{H}^{5 / 4}}
$$

dans lesquelles $n=$ nombre de révolution par minute, et où $Q$ doit être exprimé en $\mathrm{m}^{3} / \mathrm{s}$ et $\mathrm{H}$ en mètres.

$\omega$ se substituant a $n$ dans l'expression, il a paru opportun de désigner ce nombre par le symbole $\omega$, par analogie à $n_{s}$, et de l'appeler rapidité spécifique pour le différencier de vitesse spécifique, le mot rapidité ayant l'avantage de ne pas caractériser une dimension :

$$
\text { Donc : } \quad \omega_{s}=\frac{Q^{1 / 2}}{(g \mathrm{H})^{\mathrm{a} / 4}}=\text { rapidité spécifique }
$$

On a sensiblement :

$$
\omega_{s}=0,0190 n_{*}=0,0052 m_{B_{1}}
$$




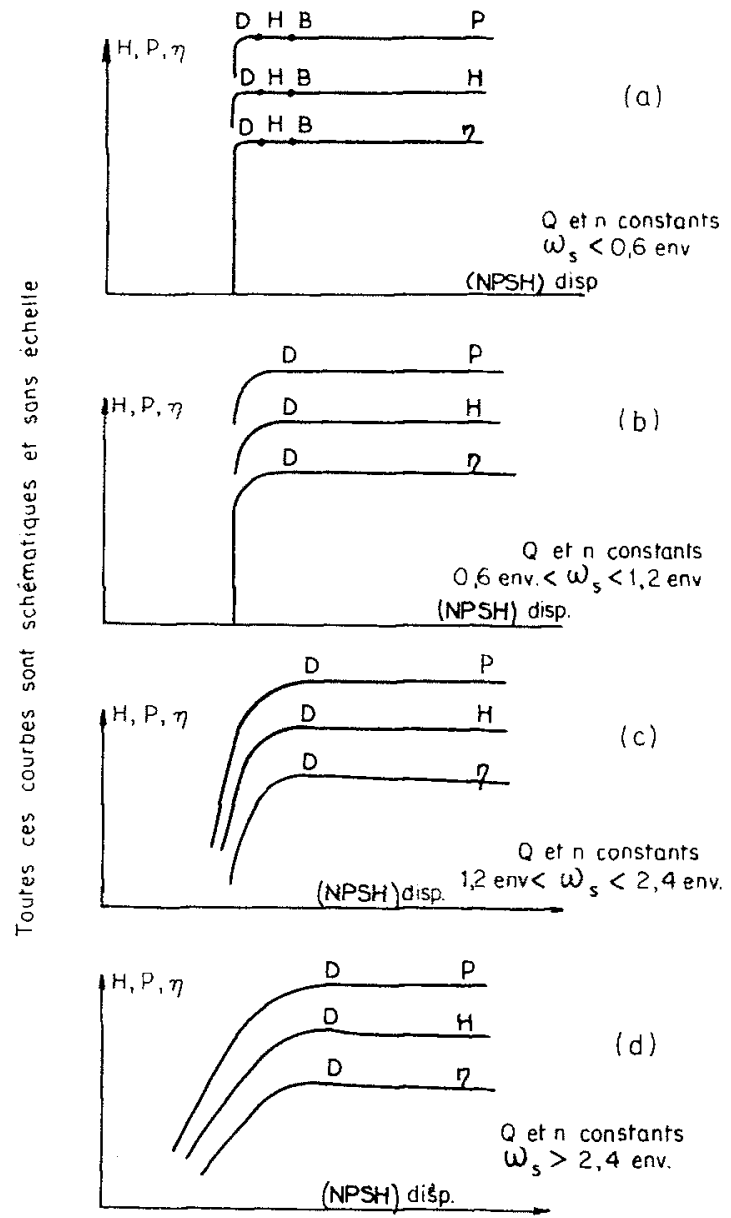

FIG. 4

$\omega_{8}<0,6$ environ, pour fixer les idées, sans que ce chiffre ait une quelconque valeur.

$\mathrm{Si}$ l'on fait décroître progressivement $(\mathrm{NPSH})_{\text {disp. }}$ à partir d'une valeur suffisamment élevée, $\mathrm{H}, \mathrm{P}$, et $\eta$ restent constants jusqu'à ce que, pour une certaine valeur du (NPSH) disp. que nous appellerons (NPSH) ${ }_{g}$, la hauteur, la puissance et le rendement tombent simultanément à la verticale de la figure. C'est ce que l'on appelle la cavitation globale, d'où l'indice $g$.

La chute se fait ici brusquement, presque sans transition.

Les figures $4 b$ et $4 c$ ont trait à une pompe centrifuge ou hélico-centrifuge de vitesse plus élevée, disons, pour fixer les idées, $0,6<\omega_{s}<2,4$.

Quand on fait baisser (NPSH) dspp, il y a chute sensiblement rectiligne comme précédemment, mais cette chute est d'autant moins verticale que la vitesse spécifique est plus élevée.

En outre, entre les horizontales de base correspondant aux grandes valeurs de (NPSH) disp. et la chute quasi rectiligne, il y a une courbe de raccordement arrondie d'autant plus importante que la vitesse spécifique est plus élevée.
Ces courbes de raccordement peuvent mème, dans certains cas, présenter une légère bosse correspondant à une légère augmentation de hauteur et de rendement.

Grosso modo, les courbes évoluent avec la vitesse spécifique de la figure $4 b$ à la figure $4 c$.

On peut encore parler de cavitation globale en prenant pour $(\mathrm{NPSH})_{g}$ la valeur du (NPSH) à l'origine de la chute quasi rectiligne; mais cette définition devient de plus en plus floue au fur et à mesure que la vitesse spécifique augmente.

Nous avons marqué de la lettre $d$ (décollement) le point où la cavitation commence à se faire sentir. Comme tout point de tangence, il est difficile à préciser.

La valeur correspondante du (NPSH) est : $(\mathrm{NPSH})_{d}$.

La figure $4 d$ a trait à des pompes hélicocentrifuges et hélice, de rapidité spécifique élevée, disons $\omega_{s}>2,4$ environ, pour fixer les idées.

Dans ce cas, la chute de $\mathrm{H}, \mathrm{P}$ et $\eta$ est progressive jusqu'au bout. On ne pent plus parler de cavitation globate.

Comme dans le cas précédent, il peut y avoir au début une légère exaltation de rendement et de hauteur.

\subsubsection{Cavitation industrielle.}

Pour avoir une définition valable dans tous les cas, et correspondant aux usages de la profession, on appelle par convention cavitation industrielle celle correspondant à une chute de hantcur de $3 \%$.

A cette cavitation correspondra l'indice $c$ (critique) (*).

La valeur de (NPSH) correspondante est (NPSH) $)_{c}$.

\subsubsection{Explication SchÉmatique de L'alidure des COURBES DE 2.2.1.}

La figure 5 a représente schématiquement la coupe d'une pompe centrifuge lente, avec un (NPSH) $)_{\text {disp. }}$ suffisamment faible pour que, à un débit voisin du débit de rendement maximal, il se forme une petite zone de cavitation à l'arrière de l'aube dans la région de l'œeillard.

(*) Nous avons hésité pour cet indice. C'est le plus employé, mais il a été utilisé avec des définitions très différentes, d'où une certaine confusion. Nous avions pensé un moment utiliser la lettre $i$ (industriel), mais elle est utilisée parfois dans la littérature anglo-saxonne pour «incipient», mot qui caractérise le tout début de cavitation. 

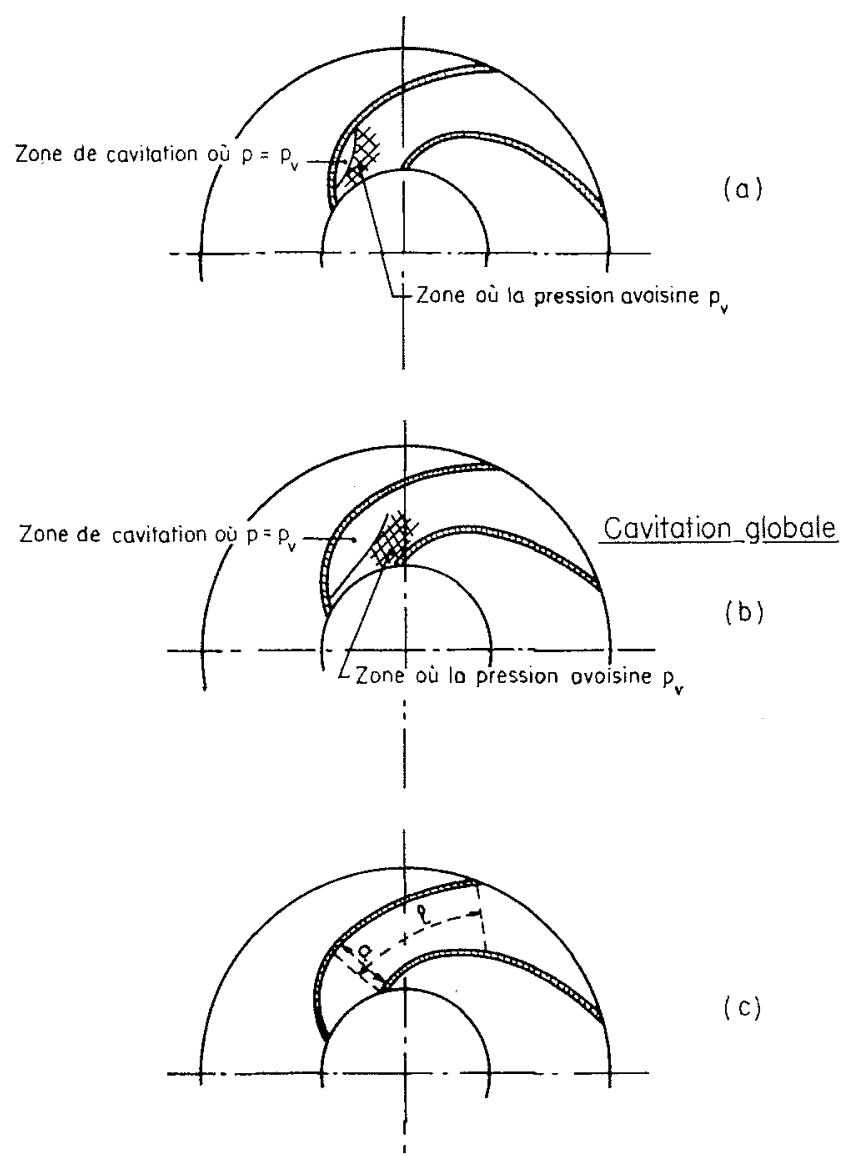

FIG. 5

Le canal étant suffisamment long, l'écoulement se reforme au-delà de la cavité et; à la sortie de la roue, n'est pas modifié par la cavitation. $H, P$, et $\eta$ restent par suite inchangés.

Cette cavitation ne se manifeste donc pas sur les courbes de fonctionnement et peut correspondre, par exemple, à un point tel que $\mathrm{M}$ de la figure $4 a$.

Quant au début de la cavitation physique, c'est-à-dire l'apparition de la moindre cavité dans l'œillard, elle passera complètement inapercue. Elle correspond par exemple au point $B$ de la figure $4 a$.

Si le $(\mathrm{NPSH})_{\text {disł. }}$ continue à décroitre, la cavitation s'étend en largeur jusqu'à ce que tout l'œillard soit à la pression $p_{v}$. Le (NPSH) correspondant est (NPSH), comme on va le voir.

En effet, quand (NPSH) diso. est maintenu à cette valeur (NPSH) $\mathrm{H}$ en faisant baisser la pression au refoulement de la pompe, le débit arrivant à l'œillard ne peut. plus augmenter puisque la charge motrice poussant le liquide dans la pompe (qui est la différence entre la charge totale à l'entrée de la pompe et la pression $p_{v}$, régnant alors dans tout l'œillard) cesse de croître, étant justement égale à (NPSH), (voir 2.1.). Le débit entrant dans la pompc reste donc égal à $Q_{g}$.

Par contre, le débit sortant de la roue, qui n'est déterminć que par les conditions à la sortie de celle-ci, augmente très passagèrement au-delà de $\mathrm{Q}_{y}$ quand $\mathrm{H}$ diminue, jusqu'à ce que la roue soit suflisamment vidée pour que les angles de sortie de l'eau se modifient et ramènent le débit sortant à $\mathrm{Q}_{g}$ (fig. $5 \mathrm{~b}$ ).

Si la longueur relative des canaux de la roue est plus faible, une zone de cavitalion d'étendue moyenne, n'occupant pas toute la largeur du canal à l'œillard, peut être néanmoins suffisante pour perturber l'écoulement à la sortie de la roue. Le canal n'est plus assez long, en effet, pour que l'écoulement se reforme inchangé, à l'aval de la zone de cavitation.

Ia perturbation à la sortic se lraduit par une modification de $H$, $P$, et $\eta$.

La valeur du (NPSH) poul laquelle la cavication commence à influer sur l'écoulement à la sortie de la roue, est celle correspondant au point $\mathrm{D}$ des figures $4 b, 4 c$, et $4 d$.

Si enfin la longueur relative des cananx est très courte ou même nulle, ce qui est le cas des roues hélices, la moindre cavitation dans l'neillard influera sur l'écoulement à la sortie de la roue et par suite sur les caractéristiques, ce qui fait que les points $D$ et $B$ seront très voisins.

D'autre part, la hauteur faite par la pompe s'annulera avant que la pression $p_{v}$ soil atteinte sur toute la largeur de l'œillard, d'où absence de cavitation globale.

Ces explications montrent, et l'expérience parait confirmer que c'est beancoup plus la longueur relative des canaux (fis. 5 c) que la rapidité spécifique qui influe sux l'allure des courbes de cavitation.

Ces deux facteurs évoluent parallelement, mais sans relation étroite, ce qui montre qu'il ne fant allacher aucune importance aux valeurs de $\omega_{g}$ indiquées plus haut pour fixer les idées.

\subsection{Modifications des courbes caractéristi- ques de fonctionnement des pompes à vitesse de rotation constante, provoquées par la cavitation.}

Si, pour une pompe donnce lournant à une vitesse donnée, au lieu de faire décroitre

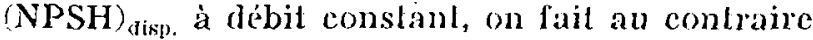
varier le débit à (NPSH) disp. constant, on peut tracer l'influence de la cavitation sur les courbes caractéristiques de fonctionnement à rilesse constante des pompes.

Ces courbes sont tracées pour des valeurs décroissantes de $(\mathrm{NPSH})_{\text {disp. }}$. soit : $(\mathrm{NPSH})_{1}$, $(\mathrm{NPSH})_{2},(\mathrm{NPSH})_{3}$, etc.

Ceci donne schématiquement les figures : $6 a$, 


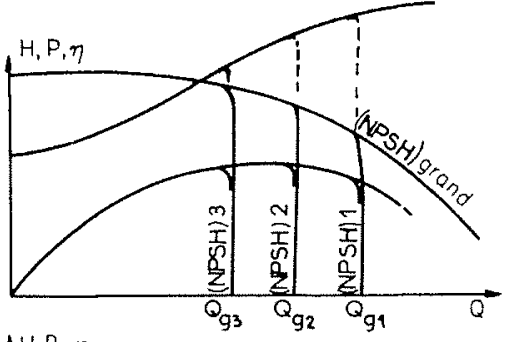

$n=c^{t e}$

$\omega_{\mathrm{s}}<0,6$ env.

(a)

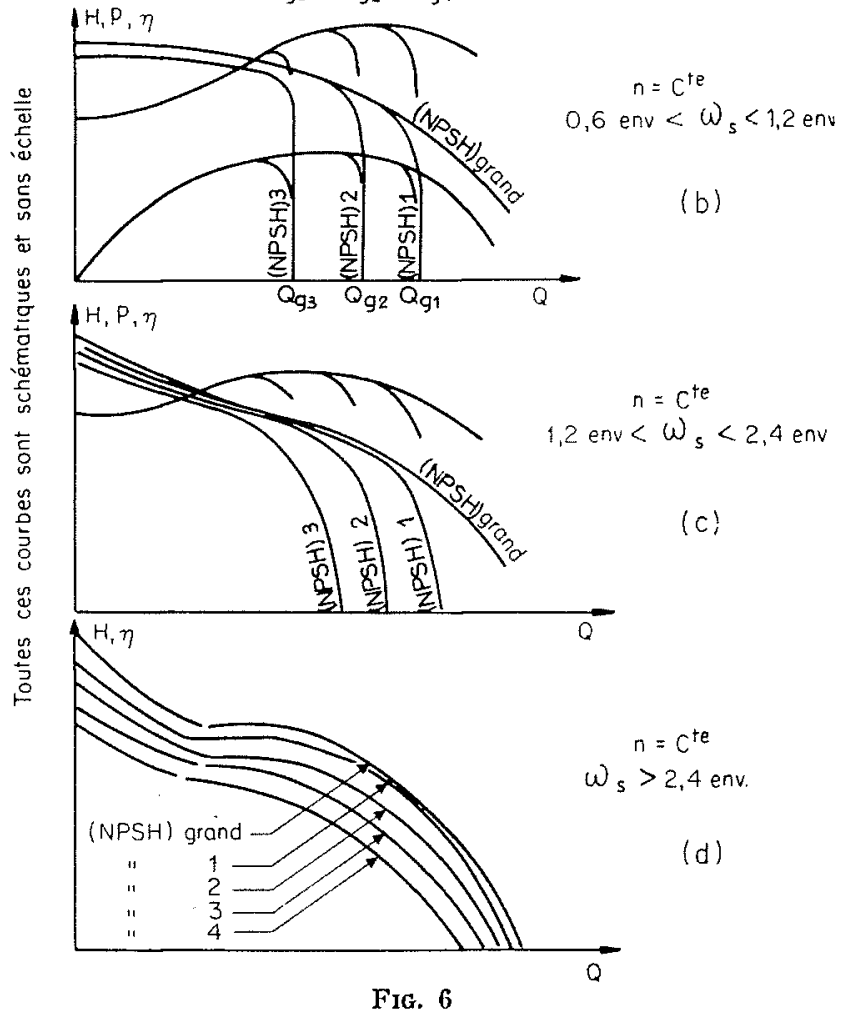

$6 b, 6 c$ et $6 d$, correspondant aux figures $4 a, 4 b$, $4 c$ et $4 d$.

Cependant, alors que dans l'examen des figures 4 , nous nous étions limités à la zone de bon rendement de la machine, les figures 5 courient toute la caractéristique hauteur-débit.

En particulier, lors des explications schématiques des figures 5, nous avions considéré ies modifications de l'écoulement dans la roue dues à la cavitation, dans des coupes, dẻveloppées ou non, perpendiculaires au plan méridien de la roue.

Or, pour des débits très inférieurs su débit de rendement maximal, la cavitation est liée à des perturbations de l'écoulement méridien. Leur influence sur les caractéristiques hauteurdébit, quoique encore mal étudiée, est retranscrite approximativement sur les courbes 6 .

\subsection{Influence du rognage d'une roue.}

La cavitation dans la roue ne dépend, nous venons de le voir, que des conditions à l'entrée de celle-ci.
On peut le montrer de façon particulièrement parlante, en rognant progressivement le diamètre extérieur d'une roue de rapidité spécifique peu élevée et en traçant les courbes hauteurdébit obtenues pour un même $(\text { NPSH) })_{\text {disp. }}$. Ceci est représenté sur la figure 7 .

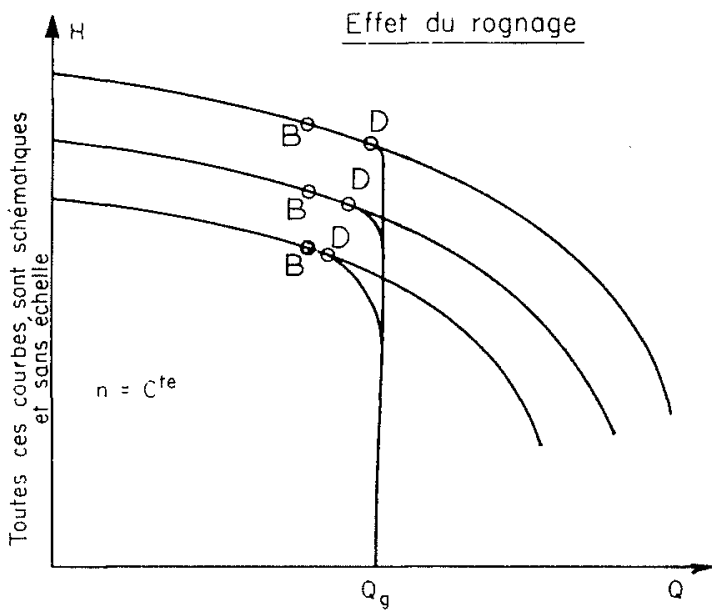

FIG. 7

Effet du rognage.

Malgré la diminution de $\mathrm{H}$, la cavitation globale se fait toujours pour le même débit $\mathrm{Q}_{g}$.

On peut supposer que le débit $Q_{b}$ correspondant au début d'apparition des bulles de cavitation (point B) reste aussi inchangé.

Quant au débit correspondant au point D, point où la courbe en cavitation décolle de la courbe sans cavitation, il se rapproche de plus en plus de $Q_{b}$ au fur et à mesure du rognage, c'est-à-dire au fur et à mesure que la longueur relative des canaux diminue. Il peut aussi en être ainsi dans une certaine mesure du débit $\mathbf{Q}_{c}$ correspondant à la cavitation industrielle.

Bien entendu, quand on augmente le diamètre extérieur d'une roue donnée, en prolongeant ses aubes, les courbes de cavitation évoluent en sens inverse.

\subsection{Caractéristiques de cavitation de l'œillard d'une roue.}

La hauteur $\mathrm{H}$ et la géométrie de la sortie de la roue n'influençant pas $(\mathrm{NPSH})_{g}$ et $(\mathrm{NPSH})_{b}$ pour un débit donné tant qu'elles n'affectent pas l'écoulement dans l'œillard, et celui-ci n'étant, par suite, fonction que du débit pour une vitesse de rotation donnée, on est conduit à caractériser les roues, et plus particulièrement leurs ceillards par les courbes $(\mathrm{NPSH})_{g}=f(\mathrm{Q})$ et $(\mathrm{NPSH})_{l}=$ $f(Q)$ à vitesse de rotation constante. 
On trace également $(\mathrm{NPSH})_{c}=f(\mathrm{Q})$, correspondant à la cavitation industriclle, mais cette dernière courbe a trait, en fait, à la totalité d'une roue et mon à son oillard seul, étant influencée plus ou moins par la sortie, comme indiqué plus haut.

Pour un œeillard donné, il peut done y avoir plusieurs courbes $(\mathrm{NPSH})_{c}=f(\mathrm{Q})$ selon le degré de rognage de la roue, ou, plus exactement, selon son diamètre extérieur.

Si l'on trace ces différentes courbes sur un même graphique, avec les (NPSH) en ordonmées et le débit en abscisse, on obtient des graphiques tels que ceux représentés schématiquement sur les figures $8 a, 8 b, 8 c$ et $8 d$, les indices $a, b, c$ et $d$ ayant trait aux mêmes gammes de vitesses spécifiques que précédemment.

La courbe $(\mathrm{NPSH})_{g}=f(\mathrm{Q})$ caractérisant la cavitation globale n'existe pas pour les vitesses spécifiques élevées.

Quant à la courbe (NPSH) $)_{b}=f(\mathrm{Q})$ caractérisant l'apparition des premières cavités, elle ne peut être, en fait, déterminée que dans les cas encore relativement rares où une observation visuelle peut être faite, grâce à des aménagements spéciaux.

Si cela est relativement facile pour des roues hélices, ce l'est beaucoup moins pour les roues centrifuges et hélicocentrifuges, sauf le cas des rouets ouverts ou de tracés anormaux ou bien si une partie de la roue est faite en plexiglas.
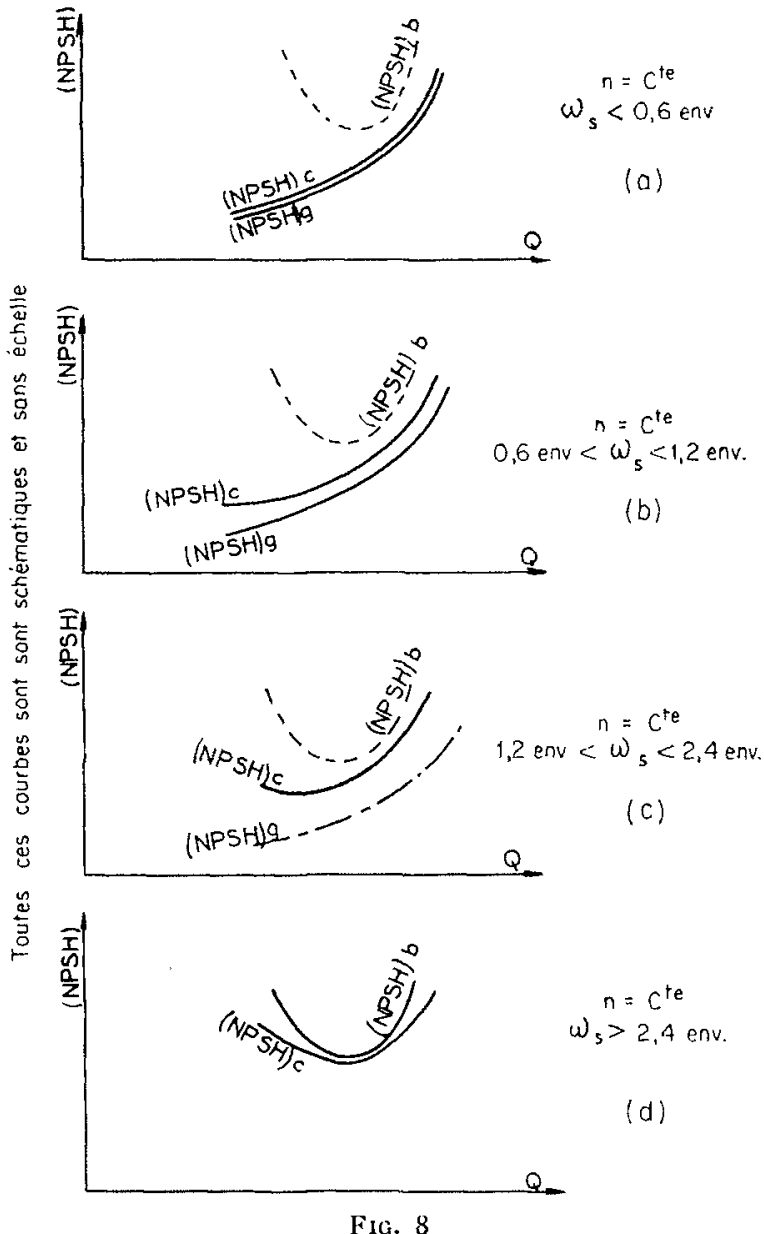

FiG. 8

\section{CHAPITRE 3}

\section{SIMILITUDE DE CAVITATION DANS LES POMPES ET ECARTS PAR RAPPORT A LA SIMILITUDE}

\subsection{Similitude de cavitation.}

\subsubsection{Similitude aU SEUIL DE la cavitation.}

Courbes $\psi_{a b}=f\left(\varphi_{a}\right)$.

Immédiatement avant l'apparition des premières bulles de cavitation, il n'y a que la phase liquide dans la pompe, et les différences de pression entre les divers points de l'intérieur de la machine suivent les lois générales de similitude des turbomachines, avec les mêmes sources d'écart par rapport à ces lois que pour les turbines (effet d'échelle, nombre de Reynolds, petits écarts dans la sinilitude géométrique, air ou gaz dissous, etc.; voir 3.3).
Par suite, toutes les grandeurs qui, dans la machine, ont la dimension d'une hauteur de colonne de liquide, varient comme le carré de toutes les vitesses d'écoulement dans la machine, elles-mêmes proportionnelles aux vitesses périphériques, pour des points de fonctionnement homologues dans la similitude.

En particulier, les grandeurs suivantes :

$$
(\mathrm{NPSH})_{b}-\frac{v_{a}^{2}}{2 g}
$$

$\left(v_{n}=\right.$ vitesse moyenne axiale d'écoulement dans l'oillard), qui représente la différence de pression entre l'entrée de la machine et le point où va apparaître la première bulle de cavitation, 
$(\mathrm{NPSH})_{b}$ et enfin $\mathrm{H}$, varient toutes comme $u_{a}{ }^{2} / 2 g$ ( $u_{a}$ étant la vitesse périphérique d'entraînement au diamètre $\mathrm{D}_{n}$ de l'œillard).

Si l'on ignore tout des dimensions de la roue, et en particulier $u_{a}$, on peut seulement faire intervenir le rapport de (NPSH) o et de $\mathrm{H}$.

soit : $\frac{(\mathrm{NPSH})_{b}}{\mathrm{H}}=\sigma_{b}$ coefficient de Thoma

expression bien connue.

$\sigma_{b}$ reste constant pour des points de fonctionnement homologues de deux pompes homothétiques ou identiques tournant à la même vitesse de rotation, ou à des vitesses de rotation différentes.

Il n'y a là évidemment aucune particularité pour les pompes par rapport aux turbines.

Cependant, le paramètre $\sigma$, en faisant intervenir $\mathrm{H}$ qui est lié presque exclusivement à la sortie de la roue et non à l'œillard, ne permet pas de passer des essais de cavitation d'une roue aux caractéristiques de cavitation d'une roue d'œillard semblable, mais de diamètre extérieur différent.

Il est donc plus avantageux, pour les constructeurs, d'écrire séparément pour l'entrée de la roue :

$$
\frac{(\mathrm{NPSH})_{b}}{u_{a}{ }^{2} / 2 g}=\mathrm{Cte}=\psi_{a b}
$$

et pour la sortie $: \frac{\mathrm{H}}{u_{r}{ }^{2} / 2 g}=\mathrm{Cte}=\psi$

$(\mathrm{NPSH})_{b}$ et $\mathrm{H}$ ne restent proportionnels entre eux que si $\left(u_{r} / u_{a}\right)=\left(\mathrm{D}_{r} / \mathrm{D}_{a}\right)$ restent eux-mêmes proportionnels entre eux pour les deux machines,

$\left(D_{r}\right.$ étant le diamètre de sortie de la roue et $u_{r}$ la vitesse d'entrainement correspondante).

Le repérage des points de fonctionnement homologues pour l'œillard étant fait le plus commodément par le débit $Q_{a}$ entrant dans la roue, le paramètre sans dimension correspondant est :

$$
\frac{\mathrm{Q}_{a}}{\mathrm{~S}_{a} u_{a}}=\frac{v_{a}}{u_{a}}=\varphi_{a}
$$

$\left(\mathrm{S}_{a}\right.$ étant l'aire utile de la section d'entrée de la roue) (voir figures $9 a$ et $9 b$ ); de même que pour l'ensemble de la pompe, on utilise parallèlement $\dot{a} \psi$ le paramètre :

$$
\varphi=\frac{\mathrm{Q}}{\mathrm{S}_{r} u_{s}}
$$

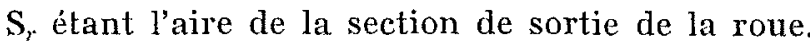
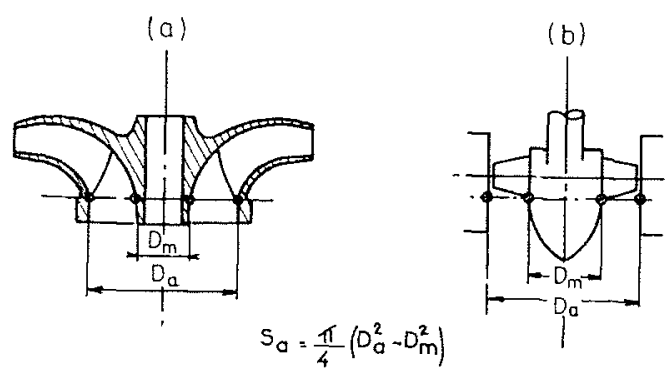

FIG. 9

Pour un weillard donné, le diagramme $\psi_{a b}=$ $f(0)_{a}$ sera donc, en théorie, indépendant des dimensions absolues de la roue et de sa vitesse de rotation.

\subsubsection{Nombres caractéristiques $\psi_{a}, \varphi_{a}, k_{a}, \pi, \omega_{s a}$} OU S ET $\omega_{s a}^{\prime}$ ou $\mathrm{S}^{\prime}$. Degré de cavitation.

3.1.2.1. $\varphi_{a}$. - Nous avons défini plus haut:

$$
\varphi_{a}=\frac{\mathrm{Q}_{a}}{\mathrm{~S}_{a} u_{a}}=\frac{v_{a}}{u_{a}}
$$

3.1.2.2. $\psi_{n}$. En appelant de façon générale :

$$
\psi_{a}=\frac{(\mathrm{NPSH})}{u_{a}^{2} / g}
$$

de mème que nous avons appelé $\psi_{a b}$ le $\psi_{a}$ ayant trait à (NPSH) $)_{b}$, nous appellerons $\psi_{a \text { alsp, }}, \psi_{a d}$, $\psi_{a r:}$ et $\psi_{a g}$ ceux ayant trait, respectivement, au (NPSH) disponible, à celui caractérisant le décollement des courbes : $(\mathrm{NPSH})_{d}$, la cavitation industrielle : $(\mathrm{NPSH})_{c}$, on la cavitation glohale : $(\mathrm{NPSH})_{g}$.

3.1.2.3. $\mathrm{K}_{a}$, - Certains expérimentateurs utilisent le coefficient :

$$
\mathrm{K}_{a}=\frac{(\mathrm{NPSH})}{v_{u}{ }^{2}} \frac{\mathrm{2} g}{2 g}
$$

On aura donc de même $K_{a b}, K_{a d}, K_{a c}, K_{a g}$.

3.1.2.4. - De même, si de façon générale:

$$
\sigma=\frac{(\mathrm{NPSH})}{\mathrm{H}}
$$

nous caractériserons de la même façon $\sigma_{\text {disp. }}, \sigma_{b}$, $\sigma_{a}, \sigma_{c}, \sigma_{g}$.

3.1.2.5. - Degré de cavitation. - Il est utile d'avoir un repérage précis du degré de cavitation quand celle-ci affecte sensiblement la courbe caractéristique hauteur-débit. 
Par convention, nous appellerons degré de cavitation d'une pompe, l'écart relatif entre la hauteur manométrique totale $\mathrm{H}$ à laquelle fonctionne réellement la pompe, telle qu'elle résulte de l'installation, et la hauteur manométrique totale $\mathrm{H}_{n}$ qu'elle donnerait pour le même débit et la mème vitesse de rotation en l'absence de toute cavitation.

$$
\text { Degré de cavitation }=d^{\circ}{ }_{c}=\frac{\mathrm{H}_{n}-\mathrm{H}}{\mathrm{H}_{n}}
$$

Pour le début de cavitation industrielle, on a donc :

$$
d^{0}{ }_{c}=0,03 \text { par convention (voir 2.2.2.). }
$$

3.1.2.6. Comparaison entre $\psi_{a}$ et $\sigma$. - Pour revenir aux avantages respectifs de $\psi_{a}$ et de $\sigma$, on peut dire ceci :

- $\omega_{a}$ intéresse plus particulièrement le constructeur, car il caractérise l'œillard d'une roue indépendamment de sa sortie et du reste de la machine. Il est de moindre intérêt pour l'utilisateur, qui ignore en général $\mathrm{D}_{a}$;

-..- $\sigma$ intéresse l'utilisateur et le projeteur, car $\sigma_{\text {disp. }}$ peut être calculé dès qu'on connaît l'installation, et sa valeur nécessaire peut être estimée dès qu'on connaît les caractéristiques de fonctionnement de la machine.

On voit cependant que, en cavitation, on peut définir deux valeurs de $\sigma$ suivant que l'on se réfère à $H$, qui généralement seul est connu, ou à $\mathrm{H}_{n}$ :

$$
\sigma=\frac{(\mathrm{NPSH})_{\mathrm{disp.}}}{\mathrm{H}} \quad \sigma^{\prime}=\frac{(\mathrm{NPSH})_{\mathrm{dis} p}}{\mathrm{H}_{n}}
$$

En l'absence de précision, il peut y avoir confusion si la cavitation est accentuée.

$$
\text { On a : } \quad \sigma^{\prime}=\sigma\left(1-d_{c}^{\circ}\right)
$$

Il faut préciser, par exemple, que les figures 4 , qui sont souvent tracées en portant $\sigma_{\text {disp. en }}$ abscisse au lieu de $(\mathrm{NPSH})_{\text {disp., }}$ ne sont valables que s'il s'agit de $\sigma^{\prime}$, c'est-à-dire de $H_{n}$ et non de $H$ au dénominateur, car, dans ce dernier cas, le $\sigma$, après avoir diminué, irait en réaugmentant pour une grande baisse de (NPSH) disp..

3.1.2.7. Vitesse spécifique d'aspiration $S$ (ou $\left.S^{\prime \prime}\right)$; rapidité spécifique d'aspiration $\omega_{s a}\left(\right.$ ou $\left.\omega_{s a}^{\prime}\right)$. - De même que la vitesse spécifique $n_{s}=$ $\left(n \mathrm{Q}^{1 / 2} / \mathrm{H}^{3 / 4}\right)$ caractérise l'aptitude d'une machine à réaliser un débit et une hauteur à une vitesse de rotation plus ou moins grande et caractérise en gros un type de roue, on a été amené à introduire la quantité :

$\mathrm{S}=\frac{n \mathrm{Q}^{1 / 2}}{(\mathrm{NPSH})^{3 / 4}}=$ vitesse spécifique d'aspiration (12)

pour caractériser :

- soit l'aptitude d'une roue à réaliser un débit donné avec le maximum de vitesse et le minimum de charge, s'il s'agit de (NPSH) ${ }_{\text {requis }}$ :

$$
(\mathrm{NPSH})_{b}, d, c \text { ou } \underset{g}{;}
$$

- soit, au contraire, la marge que l'installation procure par rapport à cette aptitude, s'il s'agit de $(\mathrm{NPSH})_{\text {disp }}$.

De même que nous avons remplacé $n_{s}$ par le nombre sans dimension :

$$
\omega_{s}=\frac{\omega^{1 / 2}}{(g \mathrm{H})^{3 / t}} \text {, rapidité spécifique }
$$

nous utiliserons pour la vitesse spécifique d'aspiration le nombre sans dimension :

$$
\begin{array}{r}
\omega_{s a}=\frac{\omega^{1 / 2}}{g^{3 / 4}(\mathrm{NPSH})^{3 / 4}} \\
\text { rapidité spécifique d'aspiration }
\end{array}
$$

On doit remarquer que $S$ et $\omega_{s t}$ font intervenir uniquement, comme $\sigma$, des paramètres extérieurs de la machine (voir 1.2) et intéressent donc l'utilisateur, et également le projeteur, avant toute considération sur le dimensionnement interne de la machine.

Le constructeur, qui doit, lui, faire intervenir ce dimensionnement, serait logiquement amené à utiliser le nombre sans dimension analogue

$$
\Omega_{n}=\frac{\varphi_{a}^{1 / 2}}{\psi_{a}^{3 / 4}}
$$

c'est-à-dire : $\quad \Omega_{n}=\frac{1}{(2 g)^{3 / 4}} \frac{u_{a} v_{t}^{1 / 2}}{(\mathrm{NPSH})^{3 / 4}}$

Pour les roues ne comportant pas d'arbre ou de moyeu d'arbre dans l'œillard on a :

$$
v_{a}=\frac{\mathrm{Q}}{\left(\pi \mathrm{D}_{a}^{2} / 4\right)} \text { et } u_{a}=\omega \frac{\mathrm{D}_{a}}{2}
$$

d'où : $\quad Q_{a}=\frac{1}{\pi^{1 / 2}(2 g)^{3 / 4}} \frac{\omega Q^{1 / 2}}{(\mathrm{NPSH})^{3 / 4}}$

c'est-à-dire : $\quad \Omega_{n}=\frac{\omega_{B a}}{\left(8 \pi^{2}\right)^{1 / 4}}$ 
Mais dans le cas général, où il peut y avoir un arbre ou un moyeu dans l'œillard, $\mathrm{S}_{a}$ diffère de $\pi \mathrm{D}_{a}{ }^{2} / 4$ (voix fig. $9 a$ et $9 b$ ).

$$
\text { En posant : } \quad \mathrm{S}_{a}=m \frac{\pi \mathrm{D}_{u}^{2}}{4}
$$

avec

$$
m \leqslant 1
$$

$(1-m)=$ degré d'obstruction de l'œillard,

il vient : $\quad \mathrm{V}_{a}=\frac{\mathrm{Q}}{m\left(\pi \mathrm{D}_{a}^{2} / 4\right)}$

d'où : $\quad \Omega_{a}=\frac{\omega_{s a}}{m^{1 / 2}\left(8 \pi^{2}\right)^{1 / 4}}$

On peut appeler :

$$
\omega_{s a}^{\prime}=\frac{\omega_{s a}}{m^{1 / 2}}=\text { rapidité spécifique }
$$

de même, on introduit :

$$
\mathrm{S}^{\prime}=\frac{\mathrm{S}}{m^{1 / 2}}=\text { vitesse spécifique }
$$

nombre ou grandeur intéressant l'utilisateur ou le projeteur, qui, même sans tenir comple des dimensions de la roue, peuvent supputer la valeur de $m$ suivant le type de construction de la pompe (par exemple machine ayant ou non un palier côté aspiration).

3.1.2.8. Relations entre $\psi_{a}, \vartheta_{a}, \mathrm{~K}_{a}, \Omega_{a}, \omega_{s a}, \omega_{s a}^{\prime}$, $\mathrm{S}, \mathrm{S}^{\prime}$ et $\sigma$. - Outre les relations (14), (16), (17) et (18) précédentes, il $\mathrm{y}$ a lieu de noter les suivantes :

$$
\begin{aligned}
\omega_{s a} & =0,0190 \mathrm{~S} \\
\omega_{s a}^{\prime} & =0,0190 \mathrm{~S}^{\prime} \\
\Psi_{a} & =\mathrm{K}_{a} \varphi_{a}^{2} \\
\Omega_{a} & =\frac{1}{\varphi_{a} \mathrm{~K}_{a}^{3 / 4}} \\
\omega_{s a} & =\frac{\omega_{s}}{\sigma^{3 / 4}} \\
\mathrm{~S} & =\frac{n_{s}}{\sigma^{3 / 4}}
\end{aligned}
$$

\subsubsection{Simllitude en cavitation.}

3.1.3.1. Considérations générales. - Quand la cavitation physique s'est développée, la similitude générale des turbomachines n'est plus théoriquement applicable, comme on le sait, que si les écoulements sont géométriquement semblables, ce qui implique des cavités géométriquement semblables et, puisque ces cavités ont une surface libre, cela implique la similitude de Froude en plus de la similitude générale des turbomachines.
Mais ceci n'est pas suffisant, car comme on le sait, il faudrait que, ce qui est contradictoire, le nombre de Reynolds soit maintenu et que, ce qui est également contradictoire, les temps de passage de l'eau dans la zone déprimée, qui conditionnent les échanges entre la phase liquide et la phase vapeur, soient aussi les mêmes.

I a question de la similitude en régime de cavitation est par suite très délicate; comme elle rentre dans le cadre des préoccupations d'un autre groupe de travail de la Commission de la Cavitation, nous ne l'examinerons pas davantage, nous réservant de revenir plus loin sur certains de ses aspects.

Signalons cependant qu'une tendance très répandue parmi les constructeurs de pompes est de faire des essais de cavitation en réalisant la même hauteur sur le modèle que sur la pompe grandeur, soit $u_{a_{\mathrm{L}}}=u_{a_{2}}$ en appelant 1 le modèle et 2 la machine grandeur.

Ceci est un moyen terme entre :

-. La similitude de Reynolds, qui impose :

$$
\frac{u_{a_{3}}}{u_{a_{3}}}=\frac{\mathrm{D}_{a_{2}}}{\mathrm{D}_{a_{1}}}
$$

En supposant que le liquide pompé ait les mêmes caractéristiques physiques pour la pompe grandeur et le modèle.

$$
\frac{u_{a_{1}}}{u_{u_{2}}}=\sqrt{\frac{\mathrm{D}_{a_{1}}}{\mathrm{D}_{a_{2}}}}
$$

- L'identité des temps de passe qui conduit à :

$$
\frac{u_{a_{1}}}{u_{a_{2}}}=\frac{\mathrm{D}_{a_{1}}}{\mathrm{D}_{t_{2}}}
$$

Le souci de faire les essais de cavitation à même hauteur pour le modèle et pour la pompe grandeur se justifie en outre pour. les raisons suivantes :

a) Certaines cavitations physiques, notamment celles apparaissant aux faibles débits, ne sont habituellement décelables, en absence de visualisation, que par le bruit perçu à l'oreille.

Or le bruit, lié à l'écrasement des bulles ou poches de cavitations dans les zones de pression, est fonction de l'importance de ces pressions.

Si les hauteurs et par suite les pressions sont trop faibles, le bruit tombe au-dessous du seuil audible et la cavitation physique en question n'est plus décelable.

Or ce bruit, souvent gênant, réapparaîtra sur la pompe grandeur. Il en est de même des vibrations.

b) Les modèles de pompe sont très souvent plus petits que les modèles réduits de turbincs (ceci pour des raisons économiques inhérentes aux deux industries). La similitude de Froude conduirait par suite à des nombres de Reynolds 
trop petits, pour les modèles de pompes, alors qu'ils sont encore suffisants pour les modèles de turbines.

3.1.3.2. Courbes $\psi_{a c}=f\left(\varphi_{1 t}\right)$ et $\psi_{a g}=f\left(\varphi_{a}\right)$. En première approximation, et. sous réserve des remarques précédentes, on admettra que la cavitation industrielle et la cavitation globale suivent les lois générales de similitude des turbomachines, ce qui justifie l'emploi des paramc̀tres :

$$
\psi_{a c}=\frac{(\mathrm{NPSH})_{0}}{u_{a}{ }^{2} / g} \text { et } \psi_{a g}=\frac{(\mathrm{NPSH})_{g}}{u_{a}^{2} / g}
$$

qui sont alors invariables dans la similitude.

Comme cela n'est qu'une approximation, on examinera séparément les écarts que- l'on a pu constater par rapport à cette similitude.

Cette façon de faire permet de tracer pour toutes les roues homothétiques entre elles la même courbe $\psi_{u c}=f\left(\varphi_{a}\right)$ et la même courbe $\psi_{a g}=\mathbf{f}\left(\varphi_{a}\right)$.

Ces courbes sont, à l'échelle près, celles des figures 8 .

3.1.3.3. Formule de Ténot. - Des essais faits à différentes vitesses sur une pompe hélice avec visualisation ont montré au professeur Ténot qu'il fallait le même écart de (NPSH) en valeur absolue en non en valeur relative par rapport au $(\mathrm{NPSH})_{c}$ pour obtenir le même degré de cavitation ou de non-cavitation.

Cela conduit à la relation :

$$
\frac{\sigma_{1 \text { disp. }}-\sigma_{\sigma_{1}}}{\sigma_{2 \text { disp. }}-\sigma_{\sigma 2}}=\frac{\mathrm{H}_{n_{2}}}{\mathrm{H}_{n_{1}}}
$$

quí est équivalente à :

$(\mathrm{NPSH})_{\text {disp. } 1}-(\mathrm{NPSH})_{c_{1}}=(\mathrm{NPSH})_{\mathrm{disp.}, 2}-(\mathrm{NPSH})_{c_{2}}$

formules dans lesquelles l'indice 1 a trait aux conditions d'exploitation de l'installation industrielle; l'indice 2 a trait aux conditions de l'essai (sur la machine mème ou sur modèle).

Cette formule (25) déduite du cas d'une même machine dont la roue a des dimensions faibles par rapport à (NPSH) disp. ne semble pas pouvoir être utilisée sans restrictions dans le cas de deux roues de dimensions très différentes ou d'une même roue de grande dimension.

\subsection{Ecarts par rapport à la similitude géné- rale.}

\subsubsection{Ecarts DUS AU MODE DE RÉalisation DES ESSAIS.}

Pour pouvoir examiner les écarts par rapport à la similitude générale, il faut d'abord commencer par discerner, pour les éliminer, ceux dus aux conditions d'essais.

\section{Citons :}

3.2.1.1. Présence de gaz dissous. - L'expérience montre en particulier que la cavitation n’apparaît pas pour le même $\psi_{a}$ s'il s'agit d'eau aérée ou désarérée.

Pour de l'eau désaérée, la cavitation apparaît plus tard, pour des $\psi_{a}$ plus faibles.

Tout se passe comme si les fines bulles d'air qui se dégagent lors de la dépression facilitaient la formation des cavités de vapeur servant de noyau à celles-ci, et ceci d'autant plus que la durée de passage (voir 3.3.2) est plus grande.

Pour toutes les pompes industrielles qui véhiculent de l'eau naturelle aérée, ce qui est la grande majorité, il convient donc que les essais de ces pompes ou de lcurs modèles soient faits également en eau aérée.

Il faudra, bien entendu, choisir un (NPSH) atsj. $_{\text {. }}$ d'essai suffisamment élevé pour que l'eau ne commence pas à dégager son air dissous avant de pénétrer dans la zone de cavitation de la machine.

Sauf indication contraire, les valeurs de $\psi_{1 k}$ auxquelles on se référera correspondront donc à des essais faits en eau aćrée.

Par contre, certaines pompes industrielles véhiculent de l'eau désaérée comme, par exemple, les pompes d'extraction de condenseur. Il est donc légitime que les essais de cavitation de ces pompes ou de leurs modèles soient faits en ean désaérée. Mais, dans ce cas, cela doit être mentionné sur les résultats d'essais.

3.2.1.2. Mode de réalisation du (NPSH). - Si on en revient à l'équation (3) paragraphe 2.1.4. et si, comme cela a presque toujours lieu, $\gamma_{\mathrm{A}}=\gamma_{0}$, on $\mathrm{a}$ :

$$
(\mathrm{NPSH})_{\mathrm{disp}}=p_{\mathrm{VA}}+\mathrm{H}_{\mathrm{A}}-\mathcal{T}_{\mathrm{A}}-p_{0}
$$

Il est rare que l'on fasse varier $p_{v}$, les essais étant faits le plus souvent en eau froide. Il faut donc faire varier $p_{\mathrm{VA}}, \mathrm{H}_{\mathrm{A}}$ ou $\mathcal{Y}_{\mathrm{A}}$ pour que le $(\mathrm{NPSH})_{\mathrm{disp}}$ varie.

a) Variation de la perte de charge à l'aspiration $\mathscr{Y}_{A}$.

Elle est obtenue le plus souvent par étranglement au moyen d'un organe approprié. Il faut prendre beaucoup de précautions pour ne pas créer de perturbations dans l'écoulement à l'entrée de la roue, ces perturbations pouvant modifier sensiblement les caractéristiques de cavitation de la roue.

C'est là une cause fréquente d'erreur.

b) Variation dans la hauteur géométrique d'aspiration $\left(\mathrm{H}_{q}\right)$.

Cette méthode n'est guère employée, car elle conduit à des dispositions d'installations compliquées. 
c) Variation de la pression totale de vapeur et de gaz $p_{\curlyvee \text { r }}$ régnant dans un réservoir fermé.

Cette méthode est très cmployée, la variation de $p_{\mathrm{VA}}$ étant obtenue facilement avec une pompe à vide.

A noter cependant que si les essais sont faits avec un vide élevé dans le réservoir, l'eau se désaère rapidement.

En outre le temps de passage de l'eau dans la zone des faibles pressions, qui s'étend alors jusque dans le réservoir, est beaucoup plus grand que dans les installations réelles. Ceci est d'autant plus vai que les essais sont faits à $u_{t_{b}}^{2} / 2 g$ faible, c'est-à-dire nécessitent un vide élevé dans le réservoir si $H_{A}=0$.

d) Utilisation d'un réservoir fermé situé en contrebas de la pompe:

Le mieux, semble-t-il, est de combiner les méthodes $b$ et $c$, en mettant le réservoir fermé à plusieurs mètres au-dessous de la pompe.

Le temps de passage de l'eau dans la zone de faible pression est alors très court et on se rapproche davantage des conditions de l'installation industrielle.

L'emploi d'un compresseur combiné avec la pompe à vide permet d'obtenir des valeurs de $(\mathrm{NPSH})_{\text {disp. }}$ depuis 0 jusqu'à plusieurs dizaines de mètres.

\section{e) Circuit fermé plein avec tube de charge.}

Dans les cas où (NPSH) risp. doit être toujours maintenu supérieur à $10 \mathrm{~m}$, il est commode d'employer un circuit fermé plein avec une simple colonne piézométrique de charge.

3.2.1.3. Indétermination de la courbe en charge. - Avant de pouvoir déterminer les points $D$ et $C$ (décollement de la courbe $H-Q$ par rapport à la courbe en charge et chute de hauteur de $3 \%$ ) il faut d'abord s'assurer qu'en augmentant la charge par rapport à la courbe de base dont on part, la courbe $H-Q$ ne varie pas en sens inverse. Il faut, en effet, partir d'une courbe qui ne varie plus quand la charge augmente. Ceci est souvent une source d'imprécision ou d'erreur, pour les grandes rapidités spécifiques.

\subsubsection{TEMPS DE PASSAGE.}

Le temps que met leau pour traverser la zone de faible pression semble avoir une grande importance, comme déjà indiqué plus haut.

Cette question, qui reste controversée, est générale et sort du cadre de ce rapport.

\subsubsection{ESSAIS D'UNE MEME POMPE A DES VITESSES} DF ROTATION DIFFÉRENTES.

Divers expérimentateurs ont constaté que $\Psi_{a c}$ diminue sensiblement quand la vitesse de rotation augmente. Sans doute y a-t-il là un effet de la réduction du temps de passage.

La formule de Ténot (voir 3.1.3.3) va dans le même sens que ces résultats expérimentaux.

On ne peut cependant pas affirmer que cette diminution est générale toutes les expériences n'étant pas concordantes à ce sujet.

Une particularité classique des essais de cavitation d'une même pompe à différentes vitesses, mais que ne met pas en défaut la similitude générale, est indiquée en 3.4 , ci-après.

\subsubsection{EsSAIS DF POMPES SEMBLABLES DE DIMEN-} SIONS DIFFÉRENTES.

Outre les écarts dus aux nombres de Reynolds et au nombre de Froude, qui sont différents pour les deux machines, il faut citer :

- rugosité relative différente pour les deux poimpes;

- écarts mineurs dans la similitude géométrique, mais pouvant avoir une grosse influence sur la cavitation (épaisseur des atubes à l'entrée), forme et acuité de l'arête d'entrée, congés de raccordement, jeux marginaux, etc.

Ceci a une grande importance pour les petites pompes $\left(\mathrm{D}_{a}<200 \mathrm{~mm}\right.$, pour fixer les idées).

\subsection{Variation du débit limite d'une pompe en fonction de sa vitesse de rotation et $\mathrm{du}$ (NPSH) disponible.}

D'après la valeur du (NPSH) disponible, une augmentation de vitesse de rotation peut, sui-

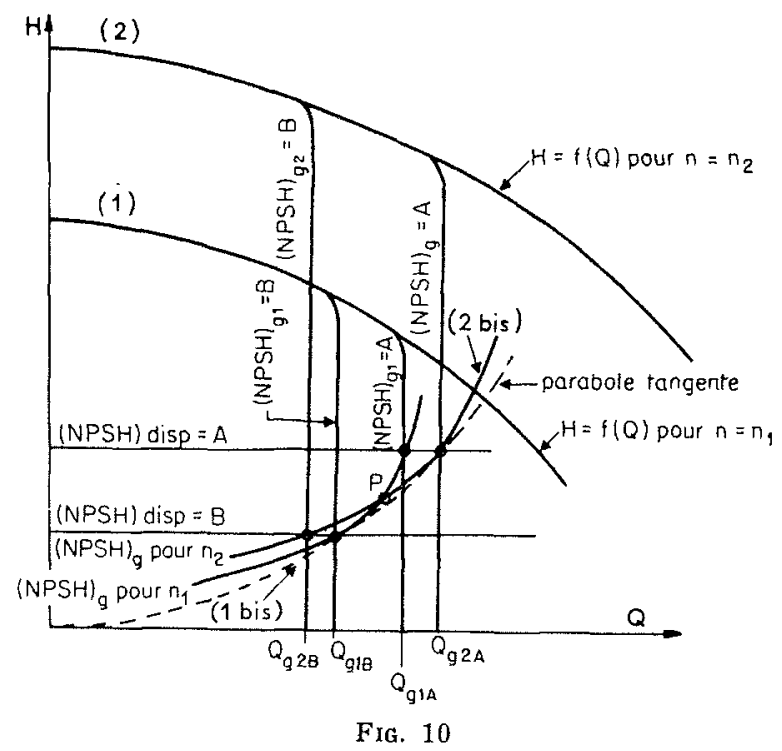


vant les cas, soit diminuer le débit atteint $(Q$. ou $\mathrm{Q}_{g}$ ), soit au contraire l'augmenter.

La figure 10 permet de le montrer.

Sur cette figure, nous avons fait figurer les courbes hauteur-débit (1) et (2) d'une pompe à deux vitesses de rolation différentes $n_{1}$ et $n_{2}>n_{1}$, ainsi que les courbes $(1 \mathrm{bis}$ ) et (2 bis) donnant $(\mathrm{NPSH})_{g}=f(\mathrm{Q})$ pour les même vitesses.
Les deux courhes ( 1 bis) et ( 2 bis) se déduisent l'une de lautre par la similitude: (NPSH), variant comme $\left(n_{2} / n_{1}\right)^{2}$ et $\mathrm{Q}$ comme $n_{2} / n_{1}$.

Ces deux courbes, incurvées vers le haut, se coupent en P. Si le (NPSH) disponible est supérieur à celui correspondant à l'ordonnée de $P$, on a $\mathrm{Q}_{g^{2}}>\mathrm{Q}_{g 1}$, comme le montre la figure.

Par contre, s'il Iui est inférieur, on a $\mathrm{Q}_{g 2}<\mathrm{Q}_{g 1}$.

\section{CHAPITRE 4}

\section{DIAGRAMMES GENERAUX - FORMULES APPROXIMATIVES}

\subsection{Diagramme général $\psi_{a}=f\left(\varphi_{u}\right)$.}

\subsubsection{PRÉSENTATION DU DIAGRAMME:}

La similitude des turbomachines étant considérée comme applicable à la cavitation sous les réserves formulées au chapitre 3, le groupe de travail «ponpes»a établi un diagramme général $\psi_{a}=f\left(\varphi_{a}\right)$ (voir fig. 11).

Ce diagramme est tracé en coordonnées logarithmiques. On $y$ a figuré le réseau des droites parallèles, $\omega_{s a}^{\prime}=$ constante caractérisant la rapidité spécifique d'aspiration corrigée. Pour que le diagramme soit plus général, on en effet choisi la rapidité spécifique d'aspiration corrigée (3.1.2.7). On y a aussi figuré quelques droites parallèles $K_{a}=$ constante et, en particulier, la droite $\mathbf{K}_{a}=1$ soit $\psi_{a}=\varphi_{u}^{2}$ c'est-à-dire

$$
(\mathrm{NPSH})=\left(v_{\alpha}^{2} / 2 g\right) \text {. }
$$

Les ordonnées mesurées par rapport à cette droite, multipliées par $u_{a}^{2} / 2 g$ soit:

$$
\frac{n_{r_{l}}^{2}}{2 g}\left(\psi_{a \text { disp. }}-\varphi_{a}{ }^{2}\right)
$$

c'est-à-dire : $\left[(\mathrm{NPSH})_{\text {disp. }}-\frac{v_{a}^{2}}{2 g}\right]$

représentent, mesurée en hauteur de liquide, la différence entre la pression moyenne dans l'œillard et la pression de vapeur.

C'est la marge disponible, avant cavitation, pour faire face aux dépressions dues aux aubes par rapport à la pression moyenne dans l'œeillard.

A partir des courbes $\psi_{a c}=f\left(\varphi_{a}\right)$ d'un grand nombre de pompes, réunies par les différents constructeurs ou expérimentateurs participant au groupe de travail, on a cherché à dégager, en les confrontant, des allures types et des zones moyennes pour ces courbes.
Ceci a été fait, notamment en vue de comparer ces résultats statistiques moyens à ce que donnent les formules simplifiées en usage ou publiées daus la littérature technique qui, comme on le verra en 4.3 , ne sont utilisables que sous certaines réserves.

I.e Groupe de travail poursuit actuellement cette confrontation.

Un examen critique est en effet nécessaire en vue d'éliminer les écarts dus aux conditions d'essai ou au fait que les resultats ont trait à des roues de dimensions ou de vitesses de rotation trop différentes.

Il serait souhaitable, dans un stade ultérieur, de ne se référer, par exemple, pour fixer les idées, qu'à des ceillards compris entre $D_{a}=$ $200 \mathrm{~mm}$ et $\mathrm{D}_{a}=300 \mathrm{~mm}$ et $\dot{a}$ des raleurs de $u_{a}{ }^{2} / 2 g$ comprises entre $10 \mathrm{~m}$ et $20 \mathrm{~m}$.

Dans la statistique du Groupe de travail, sur chaque courbe tar $=f\left(\varphi_{u}\right)$, le point correspondant au o pour lequel le rendement maximal de la pompe est atteint, est marqué d'un petit cercle et, à côté de ce point, la valeur du $\omega_{s}$ correspondant est indiquée.

\subsubsection{Principales zones DU Diagramme $\psi_{12}=$ $f\left(\varphi_{0}\right)$. Courbes types $\psi_{a c}=f\left(\varphi_{a}\right)$ :}

Pour résoudre un problème donnè : $\mathrm{Q}, \mathrm{H}, n$, $(\mathrm{NPSH})_{\text {disp. }}$, ce qui définit la vitesse spécifique d'aspiration, donc, dès que l'on a estimé $m$, la droite $\omega_{s i l}^{\prime}=$ Cte du graphique au-dessous de laquelle le point $\varsigma_{a}-\psi_{a c}$ de la pompe doit se situer, le constructeur a plus ou moins le choix du diamètre d'entrée de la roue $D_{a}$.

Si la pompe doit avoir une capacité d'aspiration élevée, pour augmenter la marge disponible donnce par l'expression

$$
\left[(\mathrm{NPSH})_{\mathrm{dikg},}-\left(v_{a^{2}}^{2} / 2 g\right)\right]
$$




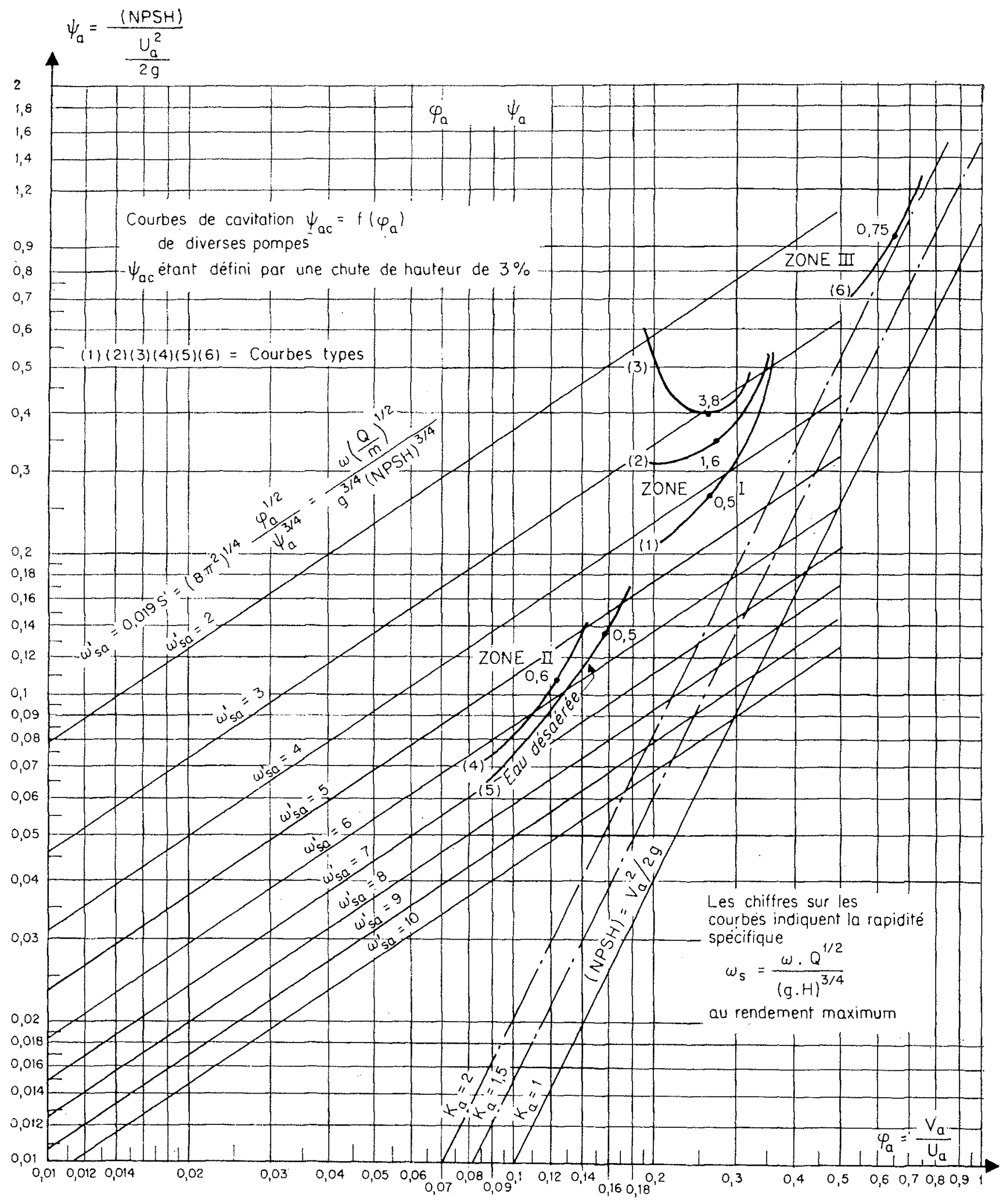

FIG. 11

Diagramme général de cavitation des pompes. 
(voir 3.1.1 et 4.1.1), on est conduit à choisir $v_{a}$ le plus faible possible, donc $D_{a}$ le plus srand possible, c'est-à-dire une valeur de of faible.

$\varphi_{a}$ varie en effet comme $1 / \mathrm{D}_{a}{ }^{3}$, car on a :

$$
\varphi_{a}=\frac{\mathrm{Q}}{(\pi / 8) m \omega \mathrm{D}_{a}^{3}}
$$

d'où :

$$
\frac{\mathrm{D}_{a}}{2}=\sqrt[3]{\frac{\mathrm{Q}}{\pi m \omega \varphi_{a}}}
$$

Comme, en l'absence de prérotation, $v_{\alpha} / u_{,}$est la tangente de l'angle de la vitesse relative de l'eau dans l'œillard au diamètre $\mathrm{D}_{n}$, avec la direction de $a_{a}$, cela conduit parallèlement à coucher les aubes à l'entrée.

On est cependant limité dans cette voie si on ne veut pas sacrifier le rendement de la roue, ou bien tomber sur des instabilités de la courbe $\mathrm{H}=f(\mathrm{Q})$ de la pompe.

Pour des tracés normaux, les roues se situent donc sur le diagramme dans une bande verticale comprise, à l'extrême, entre $\varphi_{a}=0,2$ et $\varphi_{a}=0,5$ et pratiquement entre $\varphi_{a}=0,25$ environ et $\varphi_{a}=0,35$ environ, ce qui correspond à une première zone du diagramme (Zone $I$ ).

Comme, pour un débit et une vitesse de rotation donnés et pour un degré d'obstruction de l'oillard donné, $D_{a}$ varie en raison inverse de la racine cubique de $\%_{a}$ (voir $28 \cdot$ bis) l'écart relativement faible sur on conduit à des écarts encore plus faibles sur $\mathrm{D}_{a}$. dire :

Si l'on s'en tient au cas où $m=1$, on peut

Pour un débit donné et une vitesse de rotation donnée, toutes les pompes de tracé normal, et sans moyeu dans l'oillard, ont des diamètres d'oillard du même ordre, quelle que soit leur rapidité spécifique.

Si la capacité d'aspiration prime tout, on est amené à sacrifier le rendement de la première roue et à adopter des valeurs de $\psi_{a}$ nettement plus faibles.

C'est le cas des roues de pompes d'extraction, qui se situent dans la Zone II du diagramme.

Enfin, dans certains cas, et pour des usages spéciaux ou pour des raisons particulières, des valeurs beaucoup plus grandes de $\vartheta_{\text {a }}$ ont été utilisées (œillards petits avec aubes très redressées).

Elles constituent la Zone III du diagramme.

Sur ce diagramme nous avons fait figurer, $a u$ simple titre d'illustration, quelques courbes types moyennes $\psi_{a c}=f\left(o_{i q}\right)$ qui n'ont qu'une valeur schématique.

\section{ZONE I}

Courbe 1 : Pompe centrifuge de rapidité spécifique $\omega_{s}=0,5$ environ;
Courbe 2: Pompe centrifuge de rapidité spécifique $\omega_{8}=1,6$ environ;

Courbe 3 : Pompe hélice de rapidité spécifique $\omega_{s}=3,8$ environ;

\section{ZONE II}

Courbe 4: Roue d'extraction en eau aérée :

$$
\omega_{s}=0,60 \text { environ; }
$$

Courbe 5: Roue d'extraction en eau déscérée : $\omega_{s}=0,50$ environ;

\section{ZONE III}

Courbe 6 : Roue centrifuge :

$$
\omega_{s}=0,75 \text { environ. }
$$

\subsection{Valeurs moyennes de $\omega_{s a}^{\prime}$ (ou $\left.S^{\prime}\right)$. Cour- bes $\sigma=f\left(n_{s}\right)$}

Le diagramme précédent montre qu'une même courbe caractéristique de cavitation de pompe $\psi_{a c}=f\left(\varphi_{a}\right)$ correspond à des vitesses spécifiques d'aspiration $\omega^{\prime}$ sa très différentes suivant le débit.

Pour chaque courbe, on peut déterminer trois valeurs de $\omega_{s a}^{\prime}$ :

a) La valeur maximale;

b) La valeur au rendement maximal de la pompe;

c) La valeur minimale dans les limites de débit correspondant à l'utilisation normale de la pompe.

Si l'on prend cette dernière valeur et qu'on en détermine un ordre de grandeur assez large, on peut en déduire une règle simple permettant de dégrossir rapidement un problème de pompes : par exemple :

$\omega_{s a}^{\prime}=A$ pour les tracés normaux;

$\omega_{\mathrm{s} q}^{\prime}=\mathrm{B}$ pour les tracés d'extraction.

Dans ce cas, la relation (23) ou (24) :

$$
\sigma=\left(\frac{\omega_{g}}{\left.\omega^{\prime}\right)_{s a}}\right)^{4 / 3}=\left(\frac{n_{g}}{S^{\prime}}\right)^{4 / 3}
$$

du paragraphe 3.1.2.7 devient, pour les tracés normaux

$$
\sigma=\left(\frac{1}{\mathrm{~A}}\right)^{4 / 3} \omega_{s}^{1 / 3}
$$

Ceci permet de déterminer des graphiques d'utilisation $\sigma=f\left(\omega_{s}\right)$ ou $\sigma=f\left(n_{s}\right)$ qui, en coordonnées logarithmicques, sont représentées par des droites de pente $4 / 3$ ou presque, suivant que l'on 
maintient $A$ constant ou qu'on le fait varier un peu avec $\omega_{s}$.

Le plus répandu de ces graphiques est celui des Standards de l' «Hydraulic Institute » américain que l'on trouvera retranscrit en unités métriques sur la figure 12.

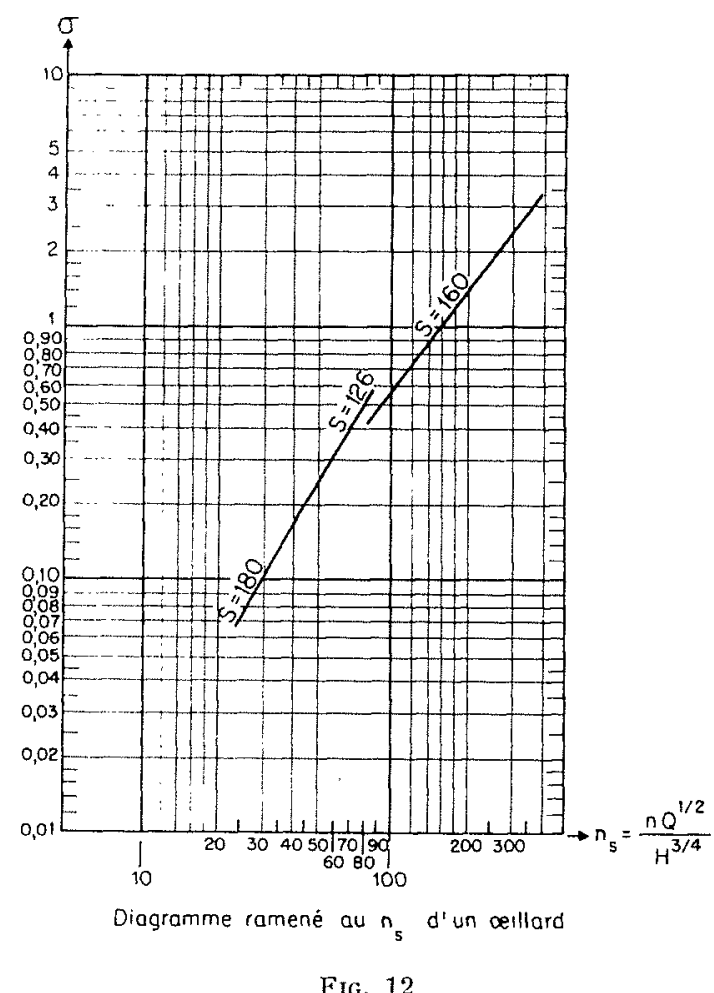

FIG. 12

Diaglamme $\sigma-n_{\star}$ déduit des standards de l'«Hydraulic Institute» américain.

Il comprend deux branches, l'une pour les pompes hélico-centrifuges et hélices, basée sur $\mathrm{S}=160$ soit $\omega^{\prime} s a=3$ environ pour $m=1$, l'autre pour les pompes centrifuges monocellulaires (avec $m=1$ ) où $S$ varie de 180 , soit $\omega_{s a}^{\prime}=3,4$ pour $n_{s}=24$, à 126 , soit $\omega^{\prime}{ }^{\prime}=2,4$ pour $n_{s}=82$.

Une comparaison avec le graphique général de la figure 11 montre que cela donne généralement une marge.

Mais cette marge est nécessaire pour tenir compte des variations de débit, donc de $\varphi_{a}$, auxquels peut être soumise la pompe et pour éliminer autant que possible la cavitation physique cachée en couvrant la courbe $\psi_{u b}=f\left(0_{0}\right)$, connue ou supposée, ceci d'autant plus que la hauteur faite par la pompe est relativement grande pour sa vitesse spécifique.

Pour les projets, il est prudent, en première approximation, de ne pas dépasser $\mathrm{S}=170$ pour les roues sans moyeu dans. I'œillard. Cela conduit, pour les roues de multi-cellulaires, avec une obstruction de l'œillard par le moyeu de l'ordre de $20 \%$ à $30 \%$, à une valeur correspondante de $S=140$ à 150 environ.

De même, pour les pompes d'extraction, en première approximation, dans les projets, il vaut mieux s'en tenir à $S \leqslant 250$ environ soit $\omega^{\prime}{ }_{s a} \leqslant 5$ environ avec obstruction de l'œillard par le moyeu de l'ordre de $15 \%$

\subsection{Formules de la forme $\psi_{a c}=K_{1} \varphi_{a}^{2}+K_{2}$}

Beaucoup d'auteurs considèrent la différence entre la pression totale à l'entrée de la machine et les cavités où règne la tension de vapeur, soit (NPSH)

-. Une dépression dans l'œillard due à la mise en vitesse $v_{a}^{2} / 2 g$ et éventuellement un peu supérieure à cette mise en vitesse moyenne par suite des survitesses locales et des petites pertes de charge, soit :

$$
\mathrm{K} \frac{v_{n}^{2}}{2 g} \quad \text { avec } \mathrm{K} \geqslant 1
$$

-- Une dépression supplémentaire due à l'action des aubes, et proportionnelle à

$$
\frac{w_{n}{ }^{2}}{2 g} \text { soit } \lambda \frac{w_{a}^{2}}{2 g}
$$

$w_{a}$ étant la vitesse relative à l'entrée des aubes au diamètre de l'œillard.

$$
(\mathrm{NPSH})_{c}=\mathbf{K} \frac{v_{n}^{2}}{2 g}+\lambda \frac{w_{u}^{2}}{2 g}
$$

$\lambda$ est variable avec $o_{n}$, augmentant rapidement de part et d'autre de la valeur de $o_{a}$ correspondant à l'angle d'entrée optimal, surtout si on détermine $\lambda$ non plus par $(\mathrm{NPSH})_{c}$, mais par $(\mathrm{NPSH})_{b}$.

En l'absence de prérotation,

$$
w_{a}^{2}=v_{a^{2}}+u_{a}^{2}
$$

d'où : $\quad(\mathrm{NPSH})_{o}=(\lambda+\mathrm{K}) \frac{v_{u}^{2}}{2 g}+\lambda \frac{u_{a}^{2}}{2 g}$

Si l'on divise les deux membres par $u_{a}{ }^{2} / 2 g$ et en ne considérant pour $\lambda$ et $\mathrm{K}$ qu'une valeur moyenne constante, $\lambda+K=K_{1}$ et $\lambda=K_{2}$, on obtient des relations de la forme :

$$
\psi_{a c}=\mathrm{K}_{1} \varphi_{a}^{2}+\bar{K}_{2}
$$

L.es valeurs de $K_{1}, K_{2}$ (ou $K$ et $\lambda$ ) proposées par divers auteurs : Pfleiderer, Gongwer, etc., sont très variables, $K_{1}$ variant de 1 à 1,5 environ et $K_{2}$ de 0,3 à 0,08 environ, croyons-nous. 
La formule de Gongwer par exemple, citée parfois donne $\mathrm{K}=1,4$ et $\lambda=\mathrm{K}_{2}=0,085$, d'où $\mathrm{K}_{1}=1,485$.

Ơn peut, en sens inverse, essayer de rechercher quelles valeurs de $K_{1}$ et de $K_{2}$ il faudrait adopter pour avoir une allure générale plus satisfaisante, par exemple tangente aux courbes (3), (1) et (6) de la figure 11.

Malheureusement, aucune valeur de $K_{1}$ et $K_{2}$ ne permet d'y parvenir, car la forme mềne de lexpression (31) ne semble pas convenir pour cela.

On peut seulement trouver des valeurs de $K_{1}$ et $K_{2}$ qui rendent ceci possible pour une plage relativement étroite de $\varphi_{a}$.

Suivant que cette plage se situe dans la zone des $\varphi_{a}$ normaux, dans celle des faibles $\varphi_{a}$ ou dans celle des $\varphi_{a}$ élevés, on aboutit ainsi à des valeurs différentes de $K_{1}$ et $K_{2}$.

Ceci explique en partie les écarts rencontrés sur ces valeurs dans la littérature.

Ces écarts sur $K_{1}$ et $K_{2}$ sont dus aussi probablement en grande partie au fait qu'il est rarement précisé quelles portions des courbes

ou

$$
\begin{aligned}
& \psi_{a b}=f\left(\varphi_{a}\right) \\
& \psi_{a c}=f\left(\varphi_{a}\right) \\
& \psi_{a b}=f\left(\varphi_{a}\right)
\end{aligned}
$$

ces formules représentatives couvrent. Enfin, il n'est pas toujours spécifié si elles ont trait à des moyennes ou à des cas extrêmes.

Le Groupe de travail ne pense done pas qu'il faille pour le moment attacher trop de valeur à ce genre de courbes.

\subsection{Relation entre $\omega_{s a}^{\prime}$ et $\omega_{s}$, c'est-à-dire entre $S^{\prime}$ et $n_{s}$ au point de rendement maximal.}

L'examen des courbes $\psi_{a c}=f\left(0_{0}\right)$ recueillies ne permet pas actuellement de déceler une loi générale de variation de la rapidité spécifique d'aspiration corrigée $\omega_{s a}^{\prime}$ de la roue en fonction de sa rapidité spécifique $\omega_{s}$.

$\omega_{s a}^{\prime}=f\left(\omega_{s}\right)$ aut point de rendement maximal des pompes.

Cependant, pour un même construcleur, s'il y a une continuité des méthodes et du style de tracé pour une large plage de $n_{s}$, une telle loi peut apparaître.

Tel constructeur, par exemple, donne pour ses tracés normaux, une variation à peu près linéaire de $\omega_{s a}^{\prime}$ entre $\omega_{s}=0,4$ et $\omega_{s}=1,3, \omega_{s a}^{\prime}$ diminuant de $40 \%$ quand $\omega_{s}$ varie de 0,4 à 1,3 .

\section{CHAPITRE 5}

\section{INFLUENCE DE LA GEOMETTRIE DE LA ROUE}

\subsection{Influence du diamètre d'œillard $\mathbf{D}_{t i}$.}

Nous avons signalé cette influence au paragraphe 4.1 . Les variations de $\mathrm{D}_{a}$ entraînent, rappelons-le, celles de $\varphi_{a}$ et par suite celles de l'angle des aubes à l'entrée qui doit s'adapter à $\varphi_{a}$, qui, avons-nous vu, en l'absence de prérotation et pour une répartition uniforme des vitesses axiales, donne la direction de la vitesse relative du liquide au diamètre $\mathrm{D}_{a}$.

\subsection{Influence de l'épaisseur des aubes à l'entrée.}

Nous avons signalé plus haut l'importance de cette épaisseur et de la forme de l'arête d'entrée, perpendiculairement à l'aube.

Pour préciser, si $Z$ est le nombre d'aubes, $e$ I'épaisseur de celles-ci à l'entrée et $\beta_{1}$ l'angle de l'aube à l'entrée sur le diamètre moyen $\mathrm{D}_{1}$ de l'arête d'entrée des aubes, la réduction relative de la section de passage à l'entrée des canaux par rapport à ce qu'elle serait avec des aubes infiniment minces, peut être estimée approximativement comme suit: La figure 13, qui représente schématiquement l'entrée d'un canal dans une vue pseudo-déreloppée, donne un ordre de grandeur de cette réduction relative en faisant abstraction des courbures, et en se limitant au

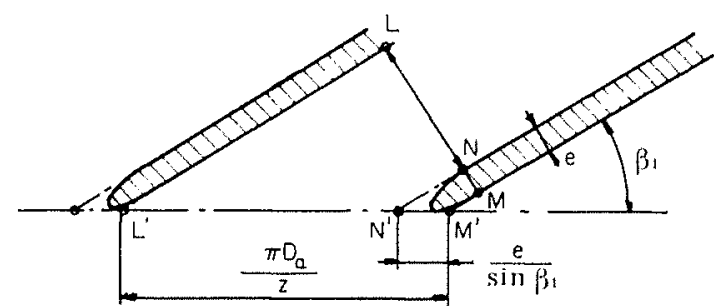

Fia. 13 
cas où l'arête d'entrée est sensiblement au dỉnmètre $\mathrm{D}_{a}$. tes :

La figure donne en effet les relations suivan-

Réduction relative :

$$
\frac{\overline{\mathrm{NL}}}{\overline{\mathrm{ML}}}=\frac{\widehat{N^{\prime} \mathrm{L}^{\prime}}}{\overline{\mathrm{M}^{\prime} \mathrm{L}}}=\frac{\left(\pi \mathrm{D}_{1} / Z\right)-\left(e / \sin \beta_{1}\right)}{\pi \mathrm{D}_{1} / \mathrm{Z}}
$$

soit : réduction relative $=1-\frac{\mathrm{Ze}}{\pi \mathrm{D}_{1} \sin \beta_{1}}$

Cette réduction entraîne une augmentation de la vitesse relative moyenne à l'entrée des canaux, d'où une augimentation de la dépression et une diminution de la capacité d'aspiration, c'est-àdire une augmentation de (NPSH) $)_{c}$ et $\psi_{a c}$.

\subsection{Influence de l'angle d'entrée moyen $\beta_{1}$ des aubes $\left(\beta_{1}\right.$ est défini en 5.2$)$.}

Cette question reste du domaine propre de chaque constructeur.

Nous signalons seulement le point suivant:

Si lon trace la ligne $\mathrm{K}_{n}=$ constante tangente à la courbe $\psi_{a c}=f\left(\varphi_{a}\right)$ d'une pompe, le $\varphi_{a} d u$ point de tangence semble correspondre plus ou moins, d'après certains expérimentateurs, à un angle de la vitesse relative, en l'absence de prérotation, voisin de l'angle de l'aube.

C'est également au voisinage de ce point que la courbe $\psi_{a b}=f\left(\wp_{a}\right)$ est en général la plus proche de la courbe $\psi_{a c}=f\left(\varphi_{a}\right)$.

Il est à noter que pour les valeurs de of audelà de ce point, c'est la face arant de l'aube qui tend à caviter, et pour des valeurs de o on deçà de ce point, c'est la face arrière de l'aube qui tend à caviter. La courbe $\psi_{a b}=f\left(\varphi_{a}\right)$, de même que les courbes (NPSH) ${ }_{b}=f(Q)$, se compose donc en fait de deux branches ayant trait chacune à l'une des faces de l'aube (branche $\mathbf{n}^{\circ} \mathbf{1}$ et branche $n^{\circ} 2$ ) respectivement (voir fig. 15).

\subsection{Influence du tracé des aubes proprement dit et du profil de l'arête d'entrée.}

Les deux branches de la courbe $\psi_{a b}=f\left(\varphi_{a}\right)$ ou $(\mathrm{NPSH})_{b}=f(\mathrm{Q})$ dont il vient d'être question en 5.3 sont plus ou moins indépendantes l'une de l'autre et l'allure parabolique de leur raccordement, représenté sur les figures 8 et la figure 15 dépend en fait de la forme de l'arête d'entrée et du tracé des aubes au voisinage de celle-ci.

Le raccordement des deux branches peut être beaucoup moins arrondi que représenté sur les figures, et même se faire de façon plus on moins anguleuse, les deux branches pouvant aller jus$q u^{\prime} \dot{a}$ se recouper dans certains cas.

Mais les influences respectives de la forme de la section droite des aubes sur l'arête d'entrée, du profil de l'arête d'entrée dans le plan méridien et du tracé de l'aube au voisinage de l'arête d'entrée, facteurs qui influent tous les trois sur les caractéristiques de cavitation, sont des questions qui restent du domaine propre de chaque constructeur.

Divers auteur's ont cherché à établir des relations faisant intervenir la géonétrie de ces tracés. Nous ne les mentionnerons que pour mémoire.

\subsection{Influence du nombre d'aubes.}

En augmentant le nombre d'aubes $Z$, on diminue le couple transmis par chaque aube au liquide pour un débit et une hauteur donnés, donc la différence de pression moyenne entre les deux cotes de l'aube. Cela va dans le sens d'une augmentation des capacites d'aspiration de la pompe.

Par contre, en augmentant le nombre d'aubes, on diminue la section de passage utile à l'entrée des canaux, par suite de l'épaisseur de celles-ci, d'où une augmentation des vitesses relatives. Cela va dans le sens d'une diminution des capacités d'aspiration de la pompe.

Ceci montre qu'il est difficile d'en déduire une loi générale.

Pour les pompes de grand $\omega_{s}$ avec des aubes peu couchées à l'entrée, divers expérimentateurs ont trouré que si, pour une même pompe avec des aubes identiques, on en diminuait le nombre au-dessous d'une certaine valeur, (NPSH) $)_{\text {ax }}$ points successifs de rendement maximal allait en augmentant, c'est-à-dire que la capacité d'aspiration augmentait.

Encore faut-il s'assurer que la diminution de $H$, et par suite l'augmentation de $n_{s}$ qui en est résultée, n'explique pas déjà en grande partie ce résultat.

Pour les pompes de petit $\omega_{s}$ et en particulier pour les pompes d'extraction dont les aubes sont très couchées à l'entrée ( $\varphi$ a petit) on perd en général sur la capacité d'aspiration quand on augmente le nombre d'aubes, ceci par suite de la diminution de la section de passage qui en résulte. 


\section{CAVITATIONS AUTRES QUE LES CAVITATIONS DANS L'CEILLARD DE LA PREMIẼRE ROUE, DANS LA ZONE DE BON RENDEMENT}

\subsection{Cavitation physique à faible débit à l'en- trée de la roue et en amont de celle-ci.}

Pour presque toutes les rapidités spécifiques, si on utilise une pompe à des hauteurs normales ou élevées (par rapport à cette rapidité spécifique) et si on diminue le débit progressivement par rapport à celui de rendement maximal, la pompe devient bruyante et ceci d'autant plus que sa rapidité spécifique est élevée.

Très souvent, et notamment pour les faibles et moyennes rapidités spécifiques, le bruit s'atténue un peu quand, en diminuant encore le débit, on arrive au débit nul.

Il est bien connu qu'au-dessous d'un certain débit, l'écoulement méridien normal est déséquilibré et est remplacé par des écoulements méridiens lels que ceux des figures $14 a$ et $14 b$.
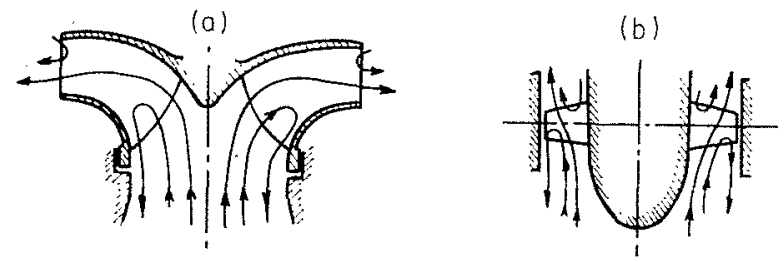

FIG. 14

En même temps la prérotation se développe à l'amont de la roue.

Ces mouvements sont générateurs de cavitations physiques locales comme a pu le montrer la visualisation.

Certains expérimentateurs ont pu mesurer ainsi les valeurs du (NPSH) ${ }_{b}$ d'apparition des cavités correspondantes et ont mis en évidence une troisième branche de la courbe $(\mathrm{NPSH})_{b}=$ $f(\mathrm{Q})$ (branche $\mathrm{n}^{\circ} 3$ ) différente des branches $\mathrm{n}^{\text {os }} 1$ et 2 de la zone de bon rendement (voir fig. 15).

L'intersection $P$ des branches 2 et 3 pourrait correspondre à la pointe de bruit. Il conviendrait de vérifier que pour un (NPSH) disponible supérieur à celui de l'ordonnée du point $P$, le bruit disparaît.

Nous ne nous étendrons pas sur l'aspect phy- sique de la cavitation de la branche 3 et sur sa localisation.

Signalons cependant que plus le débit diminue, plus les cavités tendent à se former en amont dans l'œellard et jusque dans le moyeu

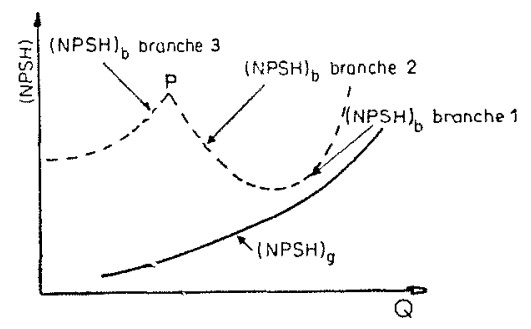

FIG. 15

de la torche de prérotation de la tuyauterie d'aspiration (ceci tout au moins pour les petites et moyennes vitesses spécifiques).

En ce qui concerne l'influence sur les courbes caractéristiques, il semble qu'au-dessous d'un certain (NPSH) disponible, la hauteur à débit nul diminue un peu.

\section{6:2. Cavitation marginale des roues hélices et des rouets ouverts et semi-ouverts.}

C'est celle qui apparaît dans la fuite entre pales et stator dans les roues hélices ou dans les roues semi-ouvertes. Elle apparait en général avant la cavitation derrière les pales.

C'est une de celles que l'on peut le plus facilement visualiser et de nombreuses photos en ont été publiées pour les roues hélices.

Elle ne semble pas influencer de facon sensible les caractéristiques de fonctionnement de la machine, mais elle est influencée par l'importance de la fuite marginale qui, elle, agit sur ces caractéristiques.

Son principal inconvénient est l'érosion, à la longue, de la ceinture fixe et de l'extrémité des pales, ce qui conduit à utiliser de préférence, pour ces parties, des mélaux résistant bien à la cavitation.

Il ne semble pas qu'il y ait de différence marquée entre les pompes et les turbines à ce sujet. 


\subsection{Cavitation dans les diffuseurs et les volutes.}

\subsubsection{CaVitation DANS LES DIFFUSEURS :}

On peut expliquer cettc cavitation schématiquement comme suit.

La pression à la sortic de la première roue d'une pompe est égale, exprimée en hauteur de liquide, à :

$$
(\mathrm{NPSH})_{\mathrm{disp} .}-\frac{v_{q}^{2}}{2 g}+\frac{\Delta p_{r}}{\gamma}
$$

$\Delta p_{r}$ étant la pression faite par la roue,

Cette pression va en diminuant quand le débit augmente, $v_{a}^{2} / 2 g$ augmentant et $\Delta p_{r}$ diminuant.

Or, quand le débit augmente très au-delà du débil normal, l'angle d'entrée de l'eau dans le diffuseur augmente, et par suite, la contraction augmentant, la section utile contractée de celuici diminue (voir fig. $16 a$ ).

Ceci, et l'augmentation de débit peuvent conduire à de très grandes vitesses dans cette seclion contractée et par suite à des dépressions atteignant l'importance de la pression disponible, d'où cavitation dans cette section.

Le débit cesse par suite d'augmenter et il $\mathrm{y}$ a chule à pic de celui-ci si on diminue la hauteur $H$ de la pompe, le diffuseur se vidant alors partiellement (voir fig. $16 \mathrm{~b}$ ).

Cette cavitation peut se produire mème en l'absence de cavilation dans la première roue.

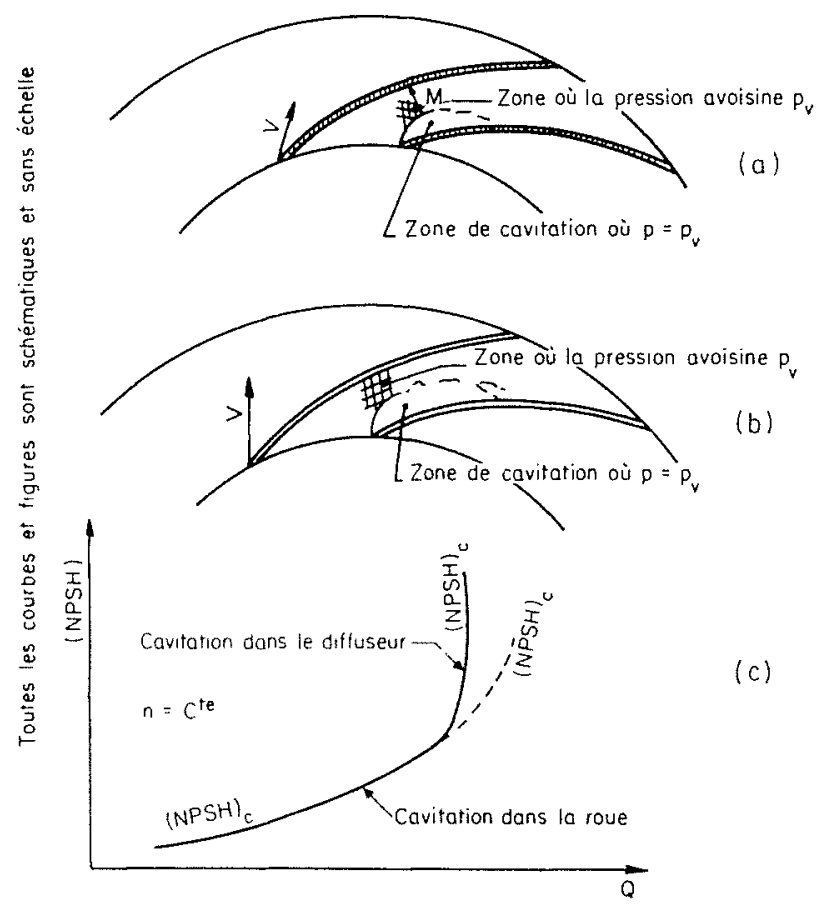

FIG. 16
Comme le débit influe ici beaucoup plus que le (NPSH) sur la pression disponible, il en résulte une remontée brutale de la courbe $(\mathrm{NPSH})_{q}=f(\mathrm{Q})$, (fig. $\left.16 \mathrm{c}\right)$.

L'examen des zones érodées de certains diffuseurs semble montrer qu'il peut y avoir aussi cavitation aux petits débits, apparemment par décollement en sens inverse derrière les aubes, à l'entrée.

\subsubsection{Cavitation aUX becs DE volutes :}

Quoique ayant quelque analogie avec les cavitations dans les diffuseurs, elle est plus rarement constatée que ces dernières, et n'affecte qu'exceptionnellement les courbes caractéristiques.

\subsection{Cavitation propre aux pompes multicel- lulaires.}

La figure 17 représente la courbe caractéristique $\mathrm{H}-\mathrm{Q}$ à vitesse constante d'une pompe multicellulaire à $m$ roues et la caractéristique faite par l'ensemble des roues sauf la première, soit les $m-1$ dernières roues.

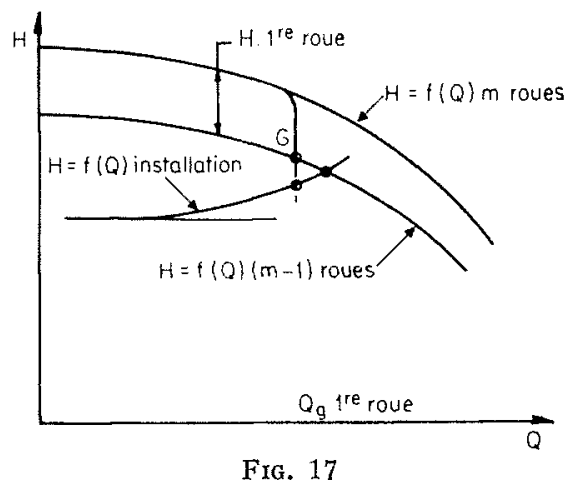

(Extraite de l'ouvrage de Stepanoff.)

Si le (NPSH) disponible est tel que la première roue soit en cavitation globale et que l'installation impose à la pompe une hauteur $\mathbf{H}$ inférieure à celle du point $G$ de la figure, le débit des $m-1$ roues de refoulement devient passagèrement supérieur à celui de la première roue.

Celle-ci cavite alors entièrement ainsi que son diffuseur; la seconde roue (et éventuellement les suivantes) cavitent alors à leur tour jusqu'à ce que les roues de refoulement ne fassent plus que le débit $\mathbf{Q}_{q}$ :

Une des conséquences, c'est que la circulation d'eau s'arrête dans la douille de la cloison médiane entre les étages 1 et 2, qui est traversée par l'arbre, ainsi que dans les bagues d'étanchéité de la première roue, et la pompe a beaucoup de chances alors de gripper. 


\section{CHAPITRE 7}

\section{FORMES DES CAVITÉS. - BRUITS ET TREPIDATIONS DÉTÉRIORATIONS DUES A LA CAVITATION}

\subsection{Forme des cavités.}

Les différents types de cavitation décrits d'après la forme des cavités ne sont pas, dans l'ensemble, différents de ceux rencontrés dans les turbines, si ce n'est qu'on ne trouve pas l'équivalent des cavitations par torches dans les aspirateurs et derrière les ogives des turbines.

Nous renverrons donc aux études faites par ailleurs à ce sujet (Voir le rapport de M. Beaufrère dans le numéro 4, 1962 de La Houille Blanche).

Quant à la liaison entre la morphologie des divers types de cavitation et leur localisation dans la pompe, le travail de synthèse correspondant reste à faire.

\subsection{Bruits et trépidations.}

Les bruits constituent un des aspects les plus gênants de la cavitation dans les pompes, mais quand ils n'affectent pas les caractéristiques de la machine, et ne sont pas accompagnés de détérioration, on est amené le plus souvent à les accepter.

Comme on le sait, ils sont dus à la fermeture brutale de cavités entraînées dans les zones de pression.

Les principales sources de bruit sont:

- la cavitation au débit partiel, même si elle n'est que physique et n'altère pas les caractéristiques (voir paragraphe 1.6) et ceci d'autant plus que la vitesse spécifique de la pompe est plus élevée;

- la cavitation au débit normal et au gros débit, et ceci d'autant plus en général que la vitesse spécifique est plus lente. Ces bruits qui apparaissent au voisinage de la cavitation critique et du point $C$ des courbes et au début de la cavitation globale, disparaissent souvent ou s'atténuent quand la cavitation globale est accentuée;

- la cavitation de diffuseur et de bec de volute, vraisemblablement tant au gros débit qu'au faible débit.
Cette question des bruits, qui est une de celles qui limitent l'emploi des pompes pour des hauteurs que l'on cherche à augmenter toujours davantage, est parmi celles dont l'étude systématique serait le plus nécessaire. Mais elle est malheureusement très complexe et d'investigation difficile.

Il en est de même des trépidations et des vibrations dues à la cavitation.

\subsection{Détériorations.}

\subsubsection{Erosions :}

Comme les bruits, elles sont dues à la fermeture brutale des cavités entraînées dans les zones de pression, et ceci dans le cas où l'écrasement se fait contre une paroi.

Si la fermeture des poches se fait au sein du liquide, il peut $y$ avoir bruit sans érosion. Cette question est du reste générale et sort du cadre de ce rapport, comme celle de la résistance des divers métaux à l'érosion.

Les principales zones d'érosion que l'on rencontre dans les pompes peuvent être figurées schématiquement sur des plans types. On peut se référer, par exemple, à celles représentées dans l'ouvrage de M. Stepanoff, Pompes centrifuges et hélices, figures XII, $10 a, b, c, d$.

Certaines sont dans les parties exemptes de cavitation comme par exemple les redresseurs de pompes hélices, mais où des poches, provenant d'une cavitation accentuée de la roue, sont entrâ̂nées par le courant. Cela peut être également le cas de roues d'extraction en pleine cavitation, provoquant l'érosion de leurs diffuseurs.

Dans ces deux cas, ce n'est pas l'organe qui cavite qui est atteint, mais celui qui est en aval.

Il serait intéressant de réunir une documentation illustrée de cas précis (point de fonctionnement, durée, etc.) et cela reste dans les ambitions du Groupe de travail.

\subsubsection{DÉformations ET Ruptures :}

Outre les érosions, les grippages et aussi les ébranlements dus aux trépidations, on pent citer comme cas de détériorations : 


\section{a) Des déformations:}

Exemple: La marche des pompes d'extraction avec une roue en pleine cavitation, l'ajustement du débit pompé au débit amené se faisant sans régulateur par le jeu de la cavitation comme cela

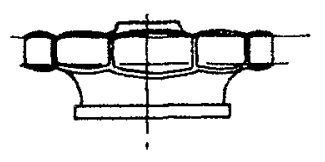

FIG. $18 a$

était fréquent jadis, avec souvent des désamorçages et réamorçages brutaux.

On a vu des cas où les flasques de la roue étaient comme soufflés entre les aubes (voir fig. $18 a$ ).

\section{b) Des ruptures :}

Exemple: La marche de pompes centrifuges à grand $n_{s}$ en cavitation accentuée à faible débit, par exemple quand elles aspirent en permanence

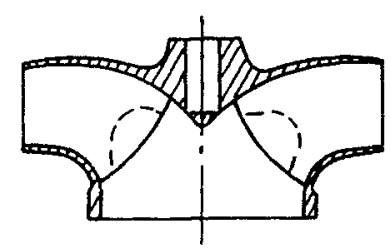

FIG. $18 b$

dans un puisard profond insuffisamment alimenté et où l'égalisation des débits se fait par cavitation. Dans certains cas, des ruptures d'aube à l'entrée ont été constatées (voir fig. $18 \mathrm{~b}$ ).

\section{POMPAGE DES LIQUIDES A PRESSION DE VAPEUR ELEVEE}

\subsection{Cas d'un liquide pur : exemple de l'eau chaude.}

\subsubsection{Constatations générales :}

Par définition, il s'agit d'un liquide dont les caractéristiques physiques : pression de vapeur, masse volumique, enthalpie, etc., à chaque température, sont nettement définies.

Le cas le plus typique et le plus fréquent est celui de l'eau chaude. Cette eau chaude est en général désaérée.

Alors que, pour l'eau froide, la masse de vapeur contenue dans une petite cavité d'un volume donné est négligeable, il n'en est plus de même en eau chaude.

Or, pour faire passer cette masse à l'état de vapeur, il a fallu lui fournir une certaine quantité de chaleux de vaporisation qui est prélevée sur l'enthalpie d'une petite quantité de liquide contiguë. Ceci nécessite un abaissement de température $\Delta \mathrm{T}$ au-dessous de celle du liquide pompé.

Cet abaissement de température, diminuant la pression de vapeur correspondante, équivaut à une augmentation du (NPSH) disponible, ce qui s'oppose à la cavitation (ou, ce qui revient au même, cela correspond à une diminution du (NPSH) requis pour ne pas dépasser ce stade de cavitation).
Le reste du liquide traverse la zone de dépression avoisinant la cavité, sans participer à cet échange, restant en quelque sorte à l'état de "surchauffe».

Ce freinage à la cavitation est d'autant plus important que la vaporisation est développée.

Pour une même pompe tournant à une même vitesse de rotation, le début de la cavitation (point B, apparition des bulles) ne sera donc pas modifié pour l'eau chaude par rapport à l'eau froide.

Par contre, pour un même degré de cavitation et plus celui-ci est élevé, plus le (NPSH) requis $_{\text {cor- }}$ respondant à ce degré de cavitation sera diminué par rapport à sa valeur en eau froide.

Ou, ce quí revient au même, pour un même (NPSH) disponible, le débit atteint pour une hauteur donnée est d'autant plus grand que la température est élevée.

La figure 19, extraite du livre de M. Stepanoff déjà cité en 7.3.1, montre à titre d'exemple les courbes de fonctionnement en cavitation d'une pompe fonctionnant à (NPSH) disp. constant et à température variable.

Un autre avantage sur l'eau froide est que, lors de la fermeture des poches entrainées dans les zones de pression, la masse de vapeur qu'elles contiennent forme coussin amortisseur, ce qui réduit les bruits, les trépidations et les érosions.

Le processus schématique décrit ci-dessus permet de se rendre compte de la complexité du 


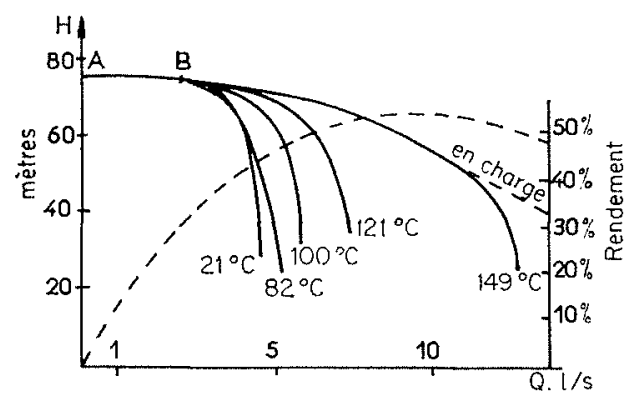

FIG. 19

(Extraite de l'ouvrage de Stepanoff.)

Pompe de $38 \mathrm{~mm}, 3740 \mathrm{tr} / \mathrm{mn}$.

Diamètre de roue : $197 \mathrm{~mm}$ (NPSH) disp. $=1,22 \mathrm{~m}$.

Le trait pointillé représente l'aspiration en charge

à $21^{\circ} \mathrm{C}$.

phénomène et du nombre de facteurs intervenant, parmi lesquels on peut citer :

- Le rapport $\frac{\text { volume vapeur }}{\text { volume liquide }}$ dans la zone de cavitation (voir 8.1.2.);

- La masse volumique de la vapeur $\imath_{v}$;

- La masse volumique du liquide $\rho_{l}$;

- La chaleur latente de vaporisation L;

- La conductibilité calorifique du fluide;

-. La chaleur spécifique du liquide $C_{l}$, qui est. la variation de l'enthalpie $i_{l}$ du liquide par degré de température $\mathrm{C}_{l}=\frac{d i_{l}}{d \mathrm{~T}}$;

- La courbe de la pression de vapeur du liquide en fonction de la température, qui donne la variation, par degré, de cette pression, soit $\frac{d p_{r}}{d \mathrm{~T}}$.
Le tableau ci-après donne :

$$
\mathrm{P}_{v}, \rho_{v}, \rho_{l}, \mathrm{~L}, \frac{d i_{l}}{d \mathrm{~T}} \text { et } \frac{d \mathrm{P}_{v}}{d \mathrm{~T}}
$$

pour l'eau aux températures $\mathrm{T}$ correspondant à $20{ }^{\circ} \mathrm{C}, 100^{\circ} \mathrm{C}, 200^{\circ} \mathrm{C}, 300^{\circ} \mathrm{C}$ et $350^{\circ} \mathrm{C}$.

Les constructeurs de pompes à eau chaude ont cherché des relations, expérimentales ou autres, reliant $(\mathrm{NPSH})_{c}$ à la température. Les courbes de correction qui en résultent sont le plus souvent à usage interne.

Citons cependant les courbes $\mathrm{BF}_{11}, \mathrm{BF}_{12}$ et $\mathrm{BF}_{17}$ des standards de l' « Hydraulic Institute » américain, courbes que nous n'avons pas reproduites.

\subsubsection{Critère thermique de cavitation (CRI- TÈRE DE STEPANOFF) :}

M. Stepanoff (voir son livre déjà cité en 7.3.1) suppose que le rapport du volume de la phase vapeur $\mathrm{V}_{v}$ au volume de phase liquide $\mathrm{V}_{l}$ intéressé par les échanges thermiques par unité de masse de liquide pompé traversant la zone de cavitation, soit $B=\left(V_{v} / V_{l}\right)$, était caractéristique du degré de cavitation $\left(\mathrm{H}_{n}-\mathrm{H}\right) / \mathrm{H}_{n}$ (voir 3.2 .5 ).

Cette hypothèse s'applique en particulier à la cavitation industrielle pour laquelle

$$
\left(\mathrm{H}_{n}-\mathrm{H}\right) / \mathrm{H}_{n}=3 \%
$$

Il suppose en outre que la quantité de chaleur nécessaire à la vaporisation de la masse de vapeur contenue dans $\mathrm{V}_{v}$ est prélevée sur l'enthalpie du liquide contenue dans $V_{l}$. Ceci revient à admettre que les volumes $V_{l}$ et $V_{v}$ se renouvellent parallèlement à la même vitesse et qu'un équilibre thermique a le temps de se produire.

\begin{tabular}{|c|c|c|c|c|c|c|c|}
\hline \multirow{2}{*}{ Caractéristique physique } & \multirow{2}{*}{ UNITS: } & \multirow{2}{*}{ SyMBole } & \multicolumn{5}{|c|}{ EAU } \\
\hline & & & à $20^{\circ} \mathrm{C}$ & à $100^{\circ} \mathrm{C}$ & à $200^{\circ} \mathrm{C}$ & à $300^{\circ} \mathrm{C}$ & à $350^{\circ} \mathrm{C}$ \\
\hline Pression de vapeur.... & bar & $p_{\eta}$ & 0,0233 & 1,012 & 15,55 & 85,9 & 165,2 \\
\hline Masse volumique du liquide....... & $\mathrm{kg} / \mathrm{m}^{3}$ & $\varphi_{l}$ & 0,998 & 0,958 & 0,866 & 0,712 & 0,572 \\
\hline Masse volumique de la vapeur..... & $\mathrm{kg} / \mathrm{m}^{3}$ & 80 & 0,0173 & 0,598 & 7,86 & 46,24 & 113,6 \\
\hline $\begin{array}{c}\text { Variation d'enthalpie du liquide par } \\
\text { degré. } \ldots \ldots \ldots \ldots \ldots \ldots \ldots \ldots \ldots \ldots \ldots \ldots\end{array}$ & $\frac{\text { cal }}{\lg \times \text { degré }}$ & $\frac{d i_{l}}{d \mathrm{~T}}$ & 1,00 & 1,00 & 1,00 & 1,3 & 2 \\
\hline 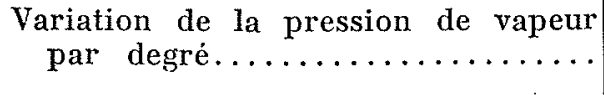 & bar/degr'é & $\frac{d p_{v}}{d \mathrm{~T}}$ & 0,0015 & 0,036 & 0,33 & 1,2 & 2,04 \\
\hline Chaleur latente de vaporisation...... & $\mathrm{cal} / \mathrm{kg}$ & $\mathrm{L}$ & 586 & 538,9 & 463,5 & 325,1 & 213,0 \\
\hline
\end{tabular}

LES VALEURS CI-DESSOUS SONT APPROCHÉES 
Dans cette hypothèse on est donc conduit à écrire :

d'où :

$$
p_{n} V_{l} \mathbf{L}=p_{l} \mathbf{V}_{l} \Delta_{i l}
$$

$$
\mathrm{B}=\frac{\mathrm{V}_{v}}{\mathrm{~V}_{i}}=\frac{p_{i} \Delta_{i l}}{p_{v} \mathrm{~L}}
$$

relation dans laquelle :

$\mathrm{B}=$ nombre sans dimension caractéristique du degré de cavitation et que M. Stepanoff a appelé Critère thermique de cavitation.

$\mathrm{V}_{v}, \mathrm{~V}_{l}, \rho_{v}, \rho_{l}, \mathrm{~L}$ ont les significations précisées cidessus en 8.1.1. et 8.1.2.

$\Delta_{i l}$ est la variation d'enthalpie correspondant à une variation déterminée de (NPSH) par abaissement de la température du liquide soit $\Delta$ (NPSH), à partir du (NPSH) pour lequel cet abaissement de température est nul, c'est-à-dire celui où le volume de la phase vapeur est encore négligeable.

Il s'agit donc théoriquement de l'écart de (NPSH) par rapport au (NPSH) ${ }_{b}$ correspondant à l'apparition des premières bulles.

Done :

$$
\Delta(\mathrm{NPSH})=(\mathrm{NPSH})_{b}-(\mathrm{NPSH})_{\mathrm{disp}}
$$

Pratiquement comme (NPSH) $)_{b}$ est rarement mesurable, M. Stepanoff le remplace par (NPSH).

Or $\Delta$ (NPSH) correspond à une variation $\Delta_{p v}$ de la pression de vapeur du liquide qui est liée à $\Delta_{i l}$ par la relation :

$$
\Delta_{i l}=\Delta_{p v} \times \frac{d_{\mathrm{T}}}{d_{p v}} \times \frac{d_{i l}}{d_{\mathrm{T}}}
$$

Si l'on admet pour simplifier que le refroidissement du liquide est uniforme dans le volume $\mathrm{V}_{t}$ et comme d'autre part :

$$
\Delta(\mathrm{NPSH})=\frac{\Delta_{p:}}{\rho_{l q}}
$$

on obtient en combinant (34), (35), (36) et (37) :

$$
\mathrm{B}=\left[\frac{\rho_{l}^{2}{ }_{i}}{\rho_{v}} \times \frac{d_{i l} / d \mathrm{~T}}{d_{l v} / d \mathrm{~T}}\right] \times\left[(\mathrm{NPSH})_{c}-(\mathrm{NPSH})_{\mathrm{disp}}\right]
$$

Si le critère B de $\mathrm{M}$. Stepanoff représente bien le degré de cavitation $\mathrm{H}_{n}-\mathrm{H} / \mathrm{H}_{n}$, son expression (38) conduit aux conclusions suivantes :

a) Pour une même pompe fonctionnant à la même vitesse de rotation avec deux liquides différents il faut, pour avoir le même degré de cavitation, que l'écart de (NPSH) par rapport à $(\mathrm{NPSH})_{b}$ supposé soit inversement proportion- nel au premier terme entre crochets de l'expression développée de $B$;

b) Pour des pompes identiques tournant à des vitesses différentes ou pour des pompes homothetiques, fonctionnant avec le même liquide (premier terme entre crochets identiques) il faut que $(\mathrm{NPSH})_{b}-(\mathrm{NPSH})_{\text {disp. }}$ reste inchangé pour avoir le même degré de cavitation et non pas $\sigma_{b}-\sigma_{\mathrm{disy}}$. inchangé, ce qui rejoint l'opinion du Professeur Tenot (voir paragraphe 3.2.3).

Bien entendu comme le fait remarquer $M$. Stepanoff, le rapport $V_{v} / V_{l}$ ne représente pas exactement le rapport géométrique des volumes de la phase vapeur et de la phase liquide dans la zone déprimée de l'œillard qui, lui, figurerait au mieux le degré de cavitation. En fonction du temps de passage plus ou moins rapide une partie du liquide échappe aux échanges thermiques : d'une part parce qu'elle n'a pas le temps de se vaporiser et traverse la zone de cavitation dans un état de surchauffe, d'autre part sans doute parce que l'abaissement de température n'a pas le temps de se communiquer à tout le liquide.

L'emploi du critère B qui a l'avantage incontestable de montrex dans quel sens influent les diverses caractéristiques physiques du liquide reste discuté par certains constructeurs et expérimentateurs et les recherches continuent dans ce domaine.

\subsubsection{Crutê̌ne de M. Gage :}

Au sujet de la cavitation des pompes à eau chaude, il est nécessaire de signaler un point important ayant trait à l'installation.

Si l'on se reporte à la figure 2 et à la relation (3), on voit qu'il faut tenir compte de l'écart entre $p_{v \mathrm{~A}}$ et $p_{v}$ et entre $\gamma_{\mathrm{A}} \gamma_{0}$.

Or, cet écart existe fréquemment pour les pompes alimentaires de chaudières, par suite des régimes transitoires de celles-ci qui font varier la température de l'eau aboutissant dans le ballon d'alimentation de la pompe.

Une marge sur le $(\mathrm{NPSH})_{\text {disp. }}$ est nécessaire pour en tenir compte.

M. Gage a établi un critère définissant cette marge, critère dans lequel intervient le rapport du volume d'eau contenu dans le ballon au volume d'eau contenu dans la tuyauterie d'alimentation de la pompe.

\subsection{Cas d'un mélange: exemple des hydro- carbures.}

S'il s'agit d'un mélange de plusieurs liquides purs ayant chacun, pris isolément, des caractéristiques physiques bien définies à chaque température, la tension de vapeur du mélange est la 
somme des pressions partielles de chaque constituant dans le liquide, qui dépend de sa tension de vapeur propre et de sa concentration, suivant la loi de Raoult.

Les hydrocarbures complexes peuvent s'écarter de cette loi.

De toute façon les parties les plus volatiles se vaporisent pour des $(\mathrm{NPSH})_{\text {disp. }}$ supérieurs à celui correspondant à la tension de vapeur du mélange.

Cependant, dans l'ensemble, il en résulte des conditions de non-cavitation, en général moins sévères que celles résultant de fonctionnement en eau froide.

L'《Hydraulic Institute» américain a publié un graphique basé sur l'expérience donnant approximativement le (NPSH) nécessaire pour les hydrocarbures courants définis par leur masse spécifique à la température de pompage, et leur tension de vapeur (voir fig. 20).

Il existe d'autres graphiques utilisés par certains constructeurs, et les recherches à ce sujet se poursuivent.

Comme, pour l'eau chaude, le (NPSH) nécessaire est en général moindre qu'une eau froide, la cavitation entraine une chute plus faible de la caractéristique $\mathrm{H}-\mathrm{Q}$, et moins de bruit et de trépidations.

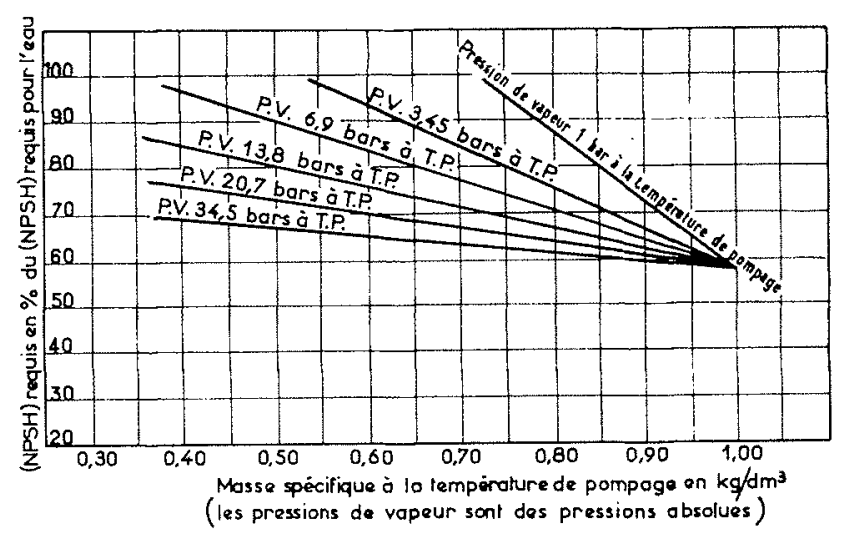

Frg. 20

Standard BF 18 de l' «Hydraulic Institute» américain. Graphique de correction de (NPSH) pour les hydrocarbures

(à ne pas utiliser pour les autres liquides).

Hydrocarbures à tension de vapeur inférieure à 1 bar à la température de pompage.

L'emploi du critère B de Stepanoff, défini de façon analogue à celui pour l'eau, a été tenté avec plus ou moins de succès. Les recherches et discussions se poursuivent à ce sujet entre constructeurs.

\section{CHAPITRE 9}

\subsection{Pompes supercavitantes.}

Il n'est pas possible de terminer ce rapport sans signaler ce type de cavitation.

Les pompes en question ont été créées pour certaines applications spéciales récentes, telles que le transfert de liquide dans les fusées, oì la réduction d'encombrement et de poids prime tout.

Elles utilisent des profils supercavitants.

Ces profils créent une poche de cavitation qui les englobe, ne restant en contact avec le liquide que par leur intrados.
Elles échappent en principe à l'érosion, les bulles entraînées venant s'écraser dans les régions de surpression à l'aval.

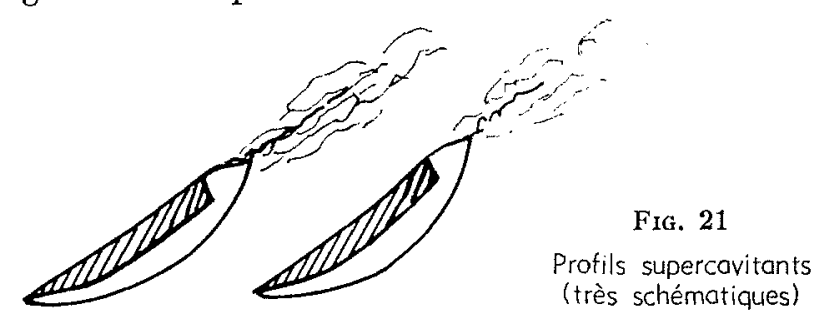

La figure 21 représente schématiquement, supposons-nous, de tels profils.

\section{CONCLUSION}

Le Groupe de travail a surtout voulu, dans ce rapport, faire le point de ce qui paraissait acquis sur le problème de la cavitation dans les pompes, afin de créer un cadre qui facilite le travail ultérieur, travail du reste déjà entrepris.

Le rapporteur du groupe regrette et s'excuse de n'avoir pu soumettre le texte définitif du rapport à ses collègues du groupe avant son impression.
Il se réserve la possibilité de diffuser ultérieurement une feuille corrective pour tenir compte de leur's observation éventuelles.

Si parfois il est allé un peu au-delà de ce qui est considéré communément comme acquis, il s'en excuse également. Mais cela aura au moins le mérite de provoquer la discussion. 